\title{
Review of pulse compression gratings for chirped pulse amplification system
}

\author{
Wenfei Zhang $\odot$, a,b,* Weijin Kong, ${ }^{\mathrm{c}}$ Guomei Wang, ${ }^{\mathrm{a}}$ Fei Xing, ${ }^{\mathrm{a}}$ \\ Fang Zhang, ${ }^{a}$ Huanian Zhang, ${ }^{\text {a,b }}$ and Shenggui $\mathrm{Fu}^{\mathrm{a}, *}$ \\ ${ }^{a}$ Shandong University of Technology, School of Physics and Optoelectronic Engineering, \\ Zibo, China \\ ${ }^{\mathrm{b}}$ Shandong Normal University, Collaborative Innovation Center of Light Manipulations and \\ Applications, Jinan, China \\ ${ }^{c}$ Qingdao University, College of Physics, Qingdao, China
}

\begin{abstract}
The ultrashort, ultrahigh intensity pulse laser has been fully developed in past three decades. Chirped pulse amplification (CPA) system plays an important role in the generation of the ultrashort, ultrahigh intensity pulse laser. Pulse compression gratings (PCGs) are the key element of CPA system and determine the performance and lifetime of the whole system. We introduce the principle of CPA system and the performance requirements of PCGs. Then the development status of PCGs, including Au-coated grating, multilayer dielectric grating (MDG), and metal MDG, is fully reviewed. Finally, the development prospect of PCGs in the future is presented. Our study is helpful for comprehensive understanding of PCGs. (c) 2021 Society of Photo-Optical Instrumentation Engineers (SPIE) [DOI: 10.1117/1.OE.60.2.020902]
\end{abstract}

Keywords: chirped pulse amplification; pulse compression gratings; diffraction efficiency.

Paper 20200812V received Jul. 7, 2020; accepted for publication Jan. 13, 2021; published online Feb. 10, 2021.

\section{Introduction}

The ultrashort, ultrahigh intensity pulse laser has attracted tremendous attentions in past three decades attributed to the specific phenomenon generated in interaction between ultrashort, ultrahigh intensity pulse laser and matter, as well as various applications. ${ }^{1-8}$ Significant efforts have been made to generate higher peak intensity, and gratifying results have been achieved. ${ }^{9-32}$ The chirped pulse amplification (CPA) system ${ }^{33,34}$ plays an important role in the generation of the ultrashort, ultrahigh intensity pulse laser. ${ }^{11-14}$ Pulse compression grating (PCG) is the most critical element in CPA system, which determines the performance and lifetime of the whole laser system. Due to the ultrahigh intensity, the PCGs require some strict performance, such as high diffraction efficiency (DE), broad bandwidth, and high laser-induced damage threshold (LIDT) ${ }^{35-37}$

Up to now, there are three main kinds of PCGs fully developed. ${ }^{35,36}$ Metal grating, usually Au-coated grating (ACG), is the original kind of PCGs used in CPA system. ACG shows high DE in a broad bandwidth. However, due to the high absorption of metal, its low LIDT limits the further applications. To advance the LIDT, multilayer dielectric grating (MDG) is developed. It is absolutely made of dielectric material, which barely absorbs the energy and shows satisfactory LIDT. MDG is the dominant PCG in CPA of picosecond laser. Its fatal restriction is the narrow bandwidth, which cannot satisfy the requirement of femtosecond laser. The hybrid grating, metal multilayer dielectric grating (MMDG), provides a promising scheme that can supply both broad bandwidth and high LIDT. Its manufacture technology is under deep investigation.

ACG, MDG, and MMDG are the main stream PCGs attributed to their excellent optical performance and easy achievement. However, there are some other designs used as PCGs, such as transmission gratings, ${ }^{38-44}$ synthetic aperture gratings, ${ }^{45-52}$ photonic crystal gratings, ${ }^{53-55}$

*Address all correspondence to Wenfei Zhang, zhangwenfei@sdut.edu.cn; Shenggui Fu, fushenggui@sdut.edu.cn 
guided mode resonant gratings, ${ }^{56-58}$ total internal reflection gratings, ${ }^{59-61}$ volume gratings,${ }^{62-64}$ connecting-layer gratings, ${ }^{65-67}$ metal mirror-based gratings, ${ }^{68}$ metal-dielectric gratings,${ }^{69-71}$ twolayer gratings, ${ }^{72-76}$ and three-layer gratings. ${ }^{77}$ Resulting from the scarce applications, we do not pay particular attentions to these gratings.

In this paper, we provide the review of the PCGs, especially the significant progress over the past five years. Our focus is on the basic principles of CPA, performance requirements of PCGs and the research progress on three different kinds of PCGs. Finally, we give the development prospect of PCGs in the future.

\section{PCGs for CPA}

\subsection{CPA System}

The peak intensity of pulse laser was seriously restrained over a long time until the CPA technique was proposed in $1985 .{ }^{23}$ The CPA system is a significant breakthrough for high-intensity laser technique and makes it possible to generate much higher intensity laser. By far, the peak intensity has been improved by 10 orders of magnitude. Strickland and Mourou, who have proposed the CPA technique, were awarded the 2018 Nobel Prize in Physics for the contribution to ultrahigh intensity laser. ${ }^{78}$

The working principle of CPA system is shown in Fig. $1{ }^{79}$ Its workflow can be decomposed as follows. (i) Pulse stretching: the low-energy, ultrashort pulse generating from oscillator is dispersed to long pulse by pulse stretcher. Its duration is generally between $100 \mathrm{ps}$ and $3 \mathrm{~ns}$, and the peak intensity is reduced by several orders of magnitude after stretching. (ii) Pulse amplification: the stretched long pulse is amplified for 10 to 100 times by laser amplifier, and it carries sufficient energy. (iii) Pulse compression: the amplified long pulse with high energy is recompressed as ultrashort pulse with the same duration to the origin pulse, consequently it shows ultrahigh peak intensity and short pulse. With the help of CPA system, the laser with petawatt level energy in picosecond can be achieved. The process assures the generation of the ultrahigh intensity laser; meanwhile, it successfully avoids the non-linear effect and element damage, which maybe aroused by high intensity.

\subsection{PCGs}

The critical element of CPA system is the PCG that stretches and compresses the pulse. The PCGs work in the high-intensity environment, and their performance directly determines the efficiency and lifetime of the laser system. The special work environment needs the PCGs

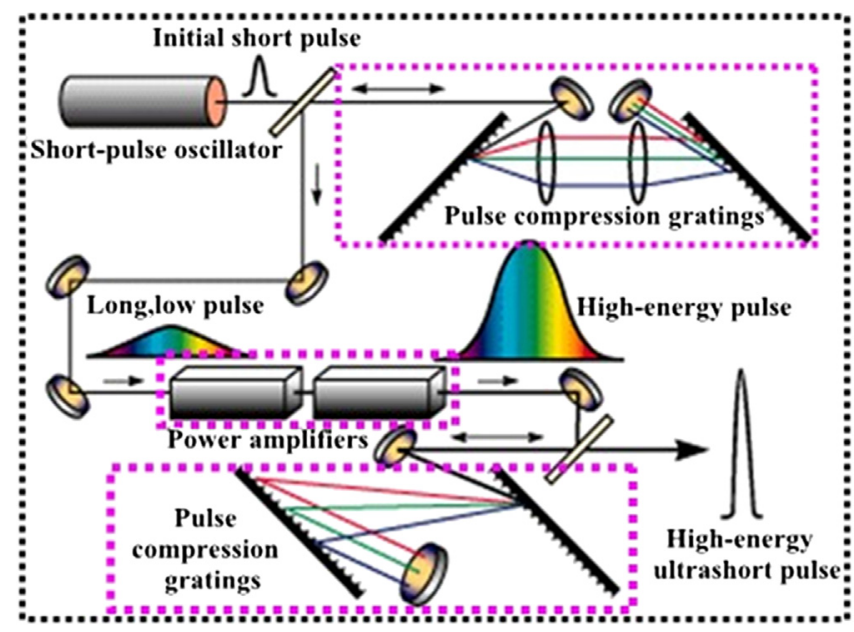

Fig. 1 Schematic of operating principle for CPA. Its workflow can be decomposed to three steps: (i) pulse stretching, (ii) pulse amplification, and (iii) pulse compression. ${ }^{79}$ 
to show the following performance. (i) High DE: the DE is an important evaluation indicator for PCGs. As shown in Fig. 1, among the process of stretching-amplification-compression, the pulse will diffract through the PCGs four times. Regardless of the scattering and absorption, the efficiency of the system is biquadrate of DE of PCG. For instance, the DE of PCG is $90 \%$, the final efficiency is $65.6 \%$. Although the DE of PCG is improved to $96 \%$, the final efficiency can reach $85 \%$. Consequently, improving the DE of PCG is significant to advance the conversion efficiency of the laser generation system. (ii) High LIDT: the PCG should tolerate the damage of the ultrahigh peak intensity laser, i.e., the high LIDT, which is the essential requirement for normal work. (iii) Broad bandwidth: as the decrease of the pulse duration, its bandwidth becomes much broader, which leads to the need for broader bandwidth of PCG. The PCG must provide high DE in sufficient bandwidth to ensure the integrality of pulse.

\section{Mathematical Background for PCGs}

The theoretical technique for analyzing the diffraction of electromagnetic waves through a grating is the basic tool to predict the diffraction behavior. In the earlier stage, the coupled-wave approach and the modal approach are commonly used. These two methods have been evidenced to be equivalent. However, they are difficult and time-consuming to achieve accurate calculations. The coupled-wave approach works well under several assumptions. To solve these problems, Moharam and Gaylord ${ }^{80}$ proposed the rigorous coupled-wave analysis (RCWA) to analyze the diffraction behavior of planar gratings. Then the RCWA was further promoted to the reflection gratings, ${ }^{81}$ arbitrary-thickness dielectric reflection gratings, ${ }^{82}$ dielectric surface-relief gratings ${ }^{83}$ planar absorption gratings, ${ }^{84}$ metallic surface-relief gratings, ${ }^{85}$ and binary gratings ${ }^{86}$ Afterward, $\mathrm{Li}^{87}$ generalized the modal method for lamellar gratings. Furthermore, the modal method was developed to multilayer modal method for arbitrary grating profile. ${ }^{88}$ The subsequent works are almost referring to these two methods.

\section{Progress on PCGs}

The PCGs have been developed for three decades, and three kinds of PCGs have been sufficiently studied. In this part, we review the PCGs in terms of structure, performance, and fabrication in detail.

\subsection{Structures of PCGs}

\subsubsection{Metal gratings}

The pioneer PCG is metal grating, which is usually fabricated as ACG. Its fabrication is typical holographic technique, which has been well developed for several decades. The grating structures are produced by the intersection of two coherent beams in a photoresponsive material. ${ }^{89-93}$ It can be decomposed into four steps, as shown in Fig. 2(a): ${ }^{94}$ (i) deposition of a uniform layer of photoresist on a flat glass substrate; (ii) holographic exposure of a standing-wave interference pattern on the photoresist layer, as shown in Fig. 2(b); ${ }^{36}$ (iii) elution of the exposed photoresist; and (iv) deposition of a thin gold film on the structure.

\subsubsection{Multilayer dielectric gratings}

To improve the low LIDT, MDGs were proposed to replace ACGs. Its structural diagram is shown in Fig. 3. It is consisted of pairs of dielectric material layers with alternating high and low refractive indices. The DE of MDG can be improved to nearly $100 \%$, and the LIDT can also be improved attributed to the low absorption of dielectric materials, which has been widely

evidenced. ${ }^{95-104}$ Its performance can be optimized through adjusting the parameters of residual layer thickness, groove depth, and the material of the surface relief. 


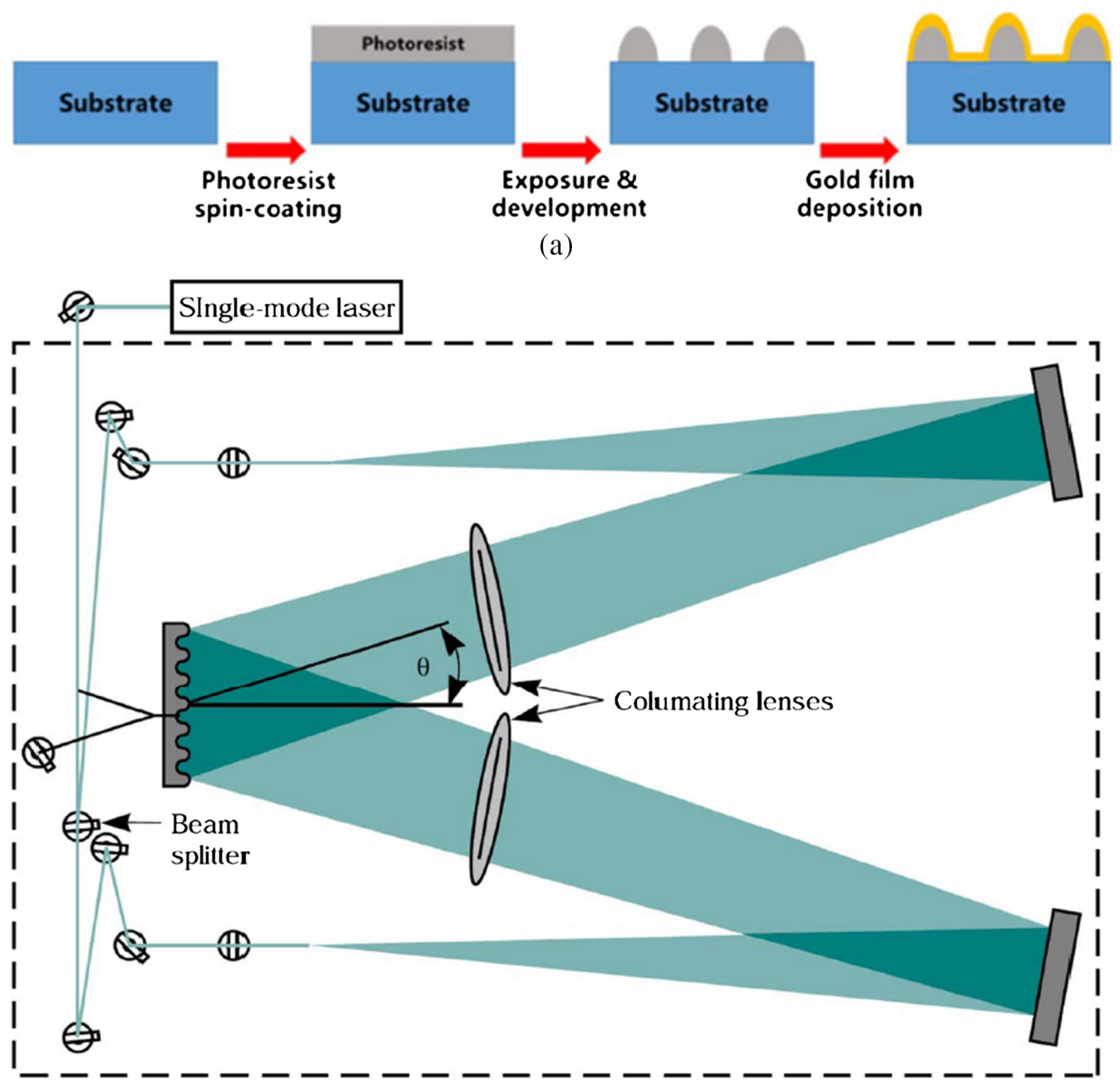

(b)

Fig. 2 The fabrication process of the ACG: (a) the four steps of ACG fabrication ${ }^{94}$ and (b) the schematic of holographic grating. ${ }^{36}$

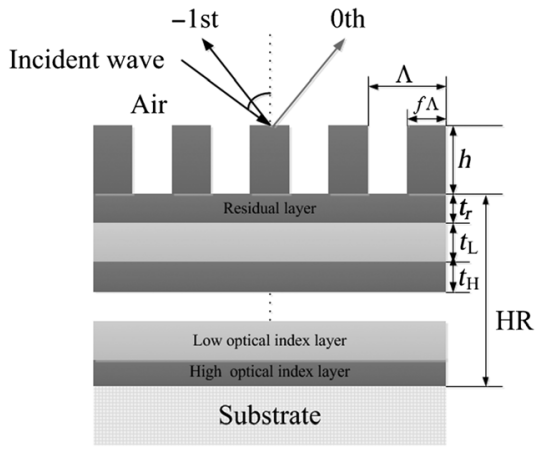

Fig. 3 Structural diagram of MDG. It was consisted of several pairs of alternating high and low dielectric films. And the groove was etched on the top layer. $h$ is the groove depth. $t_{r}$ is residual layer thickness. $\Lambda$ is the grating period. $f$ is the duty cycle. The dark and light color layers represented materials of high and low refractive indices, respectively.

\subsubsection{Metal multilayer dielectric gratings}

The key difference between MDG and MMDG is that one metal layer is inserted between the substrate and the dielectric materials in MMDG. Its structural diagram is shown in Fig. 4. It shows good LIDT and DE as that of MDG. However, the DE bandwidth can be effectively 


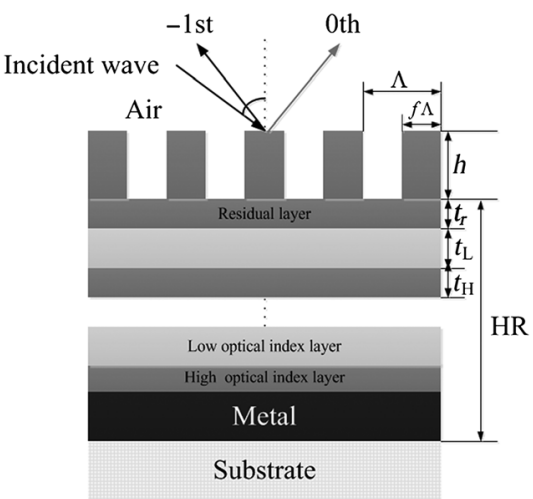

Fig. 4 Structural diagram of MMDG. It was consisted of one layer of metal and several pairs of alternating high and low dielectric films. And the groove was etched on the top layer. $h$ was the groove depth. $t_{r}$ was the residual layer thickness. $\Lambda$ was the grating period. $f$ was the duty cycle. The dark and light color layers represented materials of high and low refractive indices, respectively.

broadened. Meanwhile, the metal layer replaces some pairs of dielectric layers, which releases the stress between the whole stack and improves the stability of the structure.

\subsection{Optical Performances of PCGs}

The optical performances, such as DE, bandwidth, and central wavelength, are directly determined by the structures. The PCGs should be theoretically designed and optimized first, which is the foundation of fabrication process. In this section, we review the design progress for the PCGs.

\subsubsection{Metal gratings}

The ACG can show good DE in a broad bandwidth (close to $300 \mathrm{~nm}$ ), and the ACG is still desired element used as femtosecond compressors for high-intensity laser. ${ }^{105-108}$ However, due to the intrinsic intense absorption property of metal, its utilization is seriously restrained by its low LIDT. Therefore, the further research focuses on the improvement of LIDT. In 1995, Boyd et al. ${ }^{109}$ systematically investigated the design, fabrication, and LIDT of ACG. The fabricated ACG exhibited DE in excess of $95 \%$. They concluded that the DE of ACG prepared by e-beam evaporated coatings was higher than that of sputtering evaporation. They developed a simple theory to study the relationship between gold thickness and LIDT and systematically investigated the laser damage behavior for different pulses. Figure 5 shows that the theoretical and measured LIDT for 1053-nm pulse laser incident on a gold film. For long pulses, there was an approximately linear dependence on film thickness to $200 \mathrm{~nm}$. For short pulses, similar to that of long pulses, there was also a linear dependence on film thickness, but only to a thickness near the penetration depth. Beyond this thickness, LIDT was predicted to be independent of film thickness. The LIDT of gratings was always lower than that of uniform metal films, which did not agree with the expectation that the ACGs had close LIDT to gold films. The phenomenon maybe aroused by the field enhancement or the plasma formation.

In 1996, Stuart et al. investigated the LIDT of ACGs at 1053 and $526 \mathrm{~nm}$ for pulse durations ranging from $140 \mathrm{fs}$ to $1 \mathrm{~ns}$. It is found that the LIDT was limited to $0.6 \mathrm{~J} / \mathrm{cm}^{2}$ for the subpicosecond pulses at $1053 \mathrm{~nm}$. Figure 6 shows that the damage resulted from extremely rapid ablation and vaporization for very short pulses $(0.6 \mathrm{ps})$, and melting, flow, and resolidification for long pulses (900 ps). The heat conduction model was utilized to predict the dependence of LIDT on coating thickness and laser pulse duration. Figure 7(a) shows the theoretical and measured LIDT for gold films of various thicknesses at $1053 \mathrm{~nm}$. It was found that there was a linear dependence on film thickness up to $200 \mathrm{~nm}$ for long pulse (800 ps) and linear dependence on film thickness below the penetration depth. Beyond the penetration depth, the LIDT was 


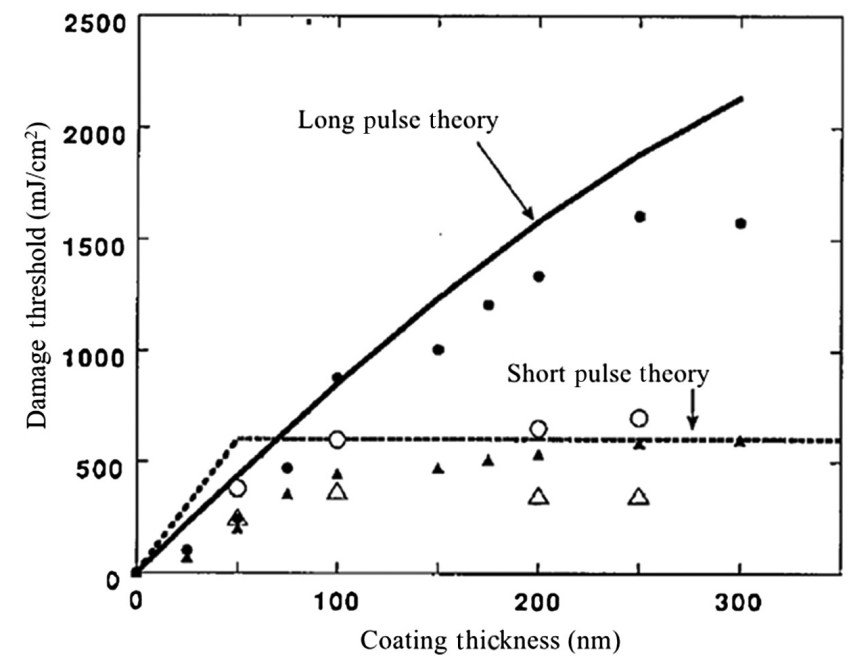

Fig. 5 Theoretical and measured LIDT for short pulse (600 fs) and long pulse ( $800 \mathrm{ps)} \mathrm{of} 1053 \mathrm{~nm}$ laser. The solid curve and the dashed curve represented the theoretical LIDT of long pulse and short pulse for a flat surface, respectively. The filled symbols showed the measured values for gold films deposited on photoresist, and the open symbols showed the measured values for gold-coated gratings. Circles were long pulse, and triangles were short pulse. ${ }^{109}$

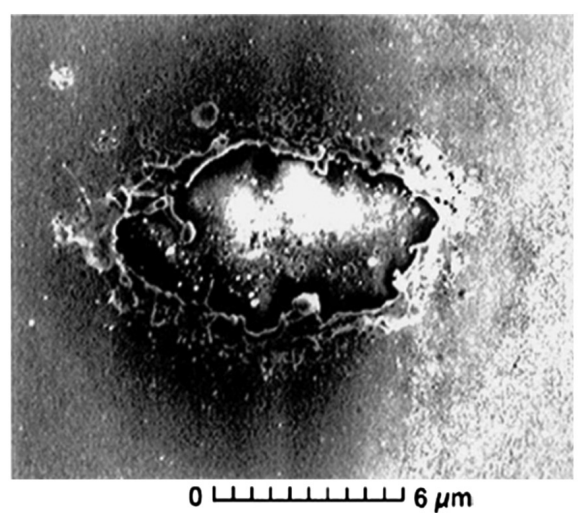

(a)

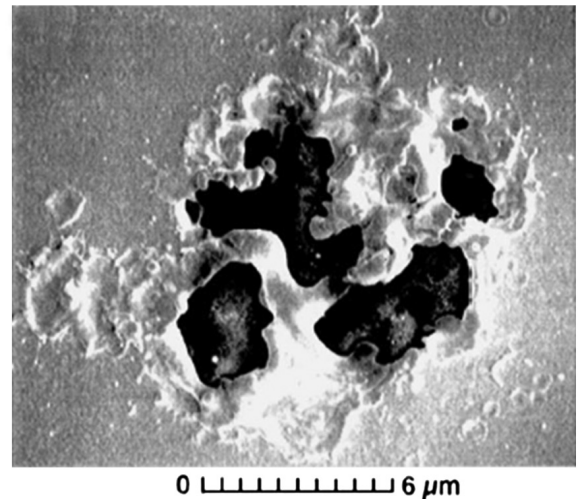

(b)

Fig. 6 Damage to gold films with $1053 \mathrm{~nm}$ pulses: (a) long pulse, $900 \mathrm{ps}$ and (b) short pulse, 0.6 ps. $^{110}$

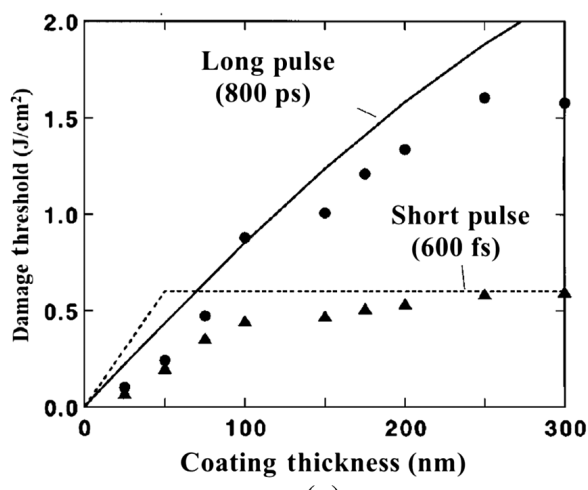

(a)

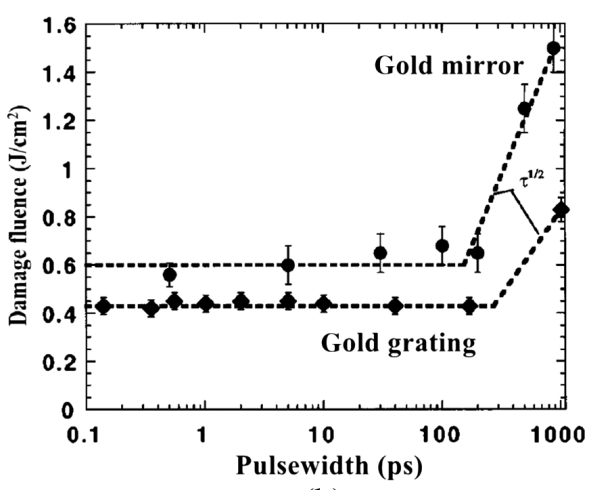

(b)

Fig. 7 Theoretical and measured LIDT for $1053 \mathrm{~nm}$. (a) LIDT for gold films with different thicknesses. Circles were long pulse ( $800 \mathrm{ps}$ ); triangles were short pulse (600 fs); and curves were the theoretical results. (b) Pulse duration dependence of LIDT of a gold grating and a gold mirror. ${ }^{110}$ 


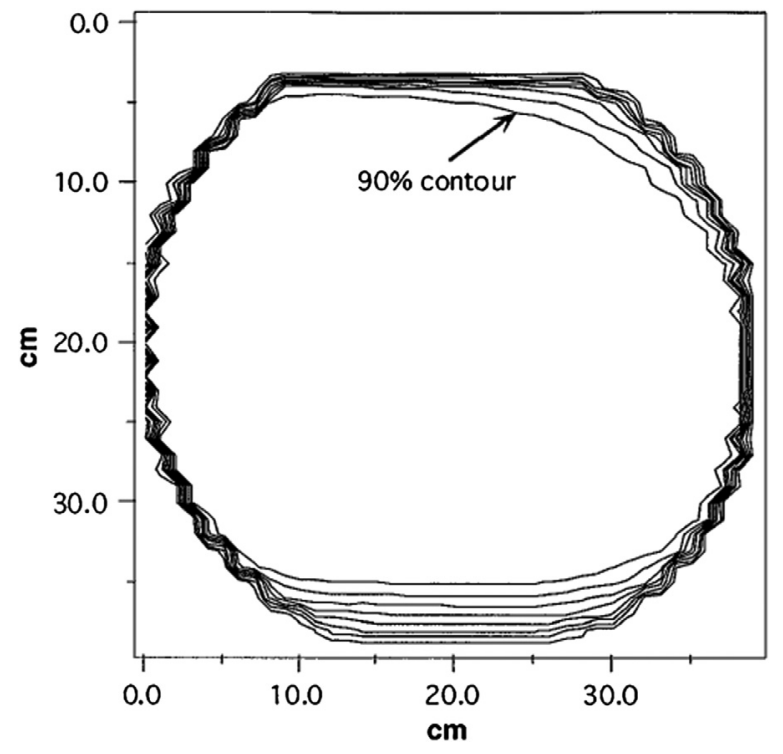

Fig. 8 Scanning photometry map of the first-order DE of a $40 \mathrm{~cm} \times 40 \mathrm{~cm} 1480 \mathrm{I} / \mathrm{mm}$ grating. The measurements were made near Littrow angle with 1047-nm TM polarization. The average DE was $94.5 \%$, neglecting the upper and lower $5 \mathrm{~cm}$ of the grating. ${ }^{111}$

independent of film thickness. The $\tau^{1 / 2}$ dependence of the LIDT on pulse duration $\tau$ was observed down to $200 \mathrm{ps}$, whereas the LIDT was nearly independent of pulse duration below $1 \mathrm{~ns}$, as shown in Fig. 7(b). The theoretical model can be well quantitative agreement with pulse duration and wavelength of experimental results. ${ }^{110}$

Britten et al. developed a holographically produced ACG. It exhibited high LIDT and 800 to $1100 \mathrm{~nm}$ wavelength range for $\mathrm{DE}>91 \%$. Maximum DE $>93 \%$ was measured for TM polarization at $1053 \mathrm{~nm}$. Figure 8 shows the DE over the whole grating with size of $40 \mathrm{~cm} \times 40 \mathrm{~cm}$. The DE was extremely uniform and $>94 \%$ over the central $90 \%$ area. The LIDT was $420 \mathrm{~mJ} / \mathrm{cm}^{2}$ for pulse durations from $100 \mathrm{ps}$ to $200 \mathrm{fs}$ at $1053 \mathrm{~nm}$. Its broad bandwidth testified the potential in stretching and compression of extremely short pulses (10 fs). However, it cannot compare with MDGs in terms of DE or LIDT. ${ }^{111}$

\subsubsection{Multilayer dielectric gratings}

Dielectric materials barely absorb any energy, so it inherently shows good LIDT. The main focus is the improvement of bandwidth with high DE through optimizing the structure parameters.

In 1995, Perry et al. reported an MDG, consisted of alternating layers of $\mathrm{ZnS}\left(n_{\mathrm{H}}=2.35\right)$ and $\mathrm{ThF}_{4}\left(n_{\mathrm{L}}=1.52\right)$, and the surface relief was etched on $\mathrm{ZnS}$. The DE of MDG was related to wavelength and polarization of incident light, the shape, and depth of the grooves. The calculations predicted that the peak DE for TE polarization was expected to $98 \%$, whereas it was $<50 \%$ for TM polarization. The DE was highly sensitive to the polarization of the incident light, which was widely confirmed in later works. The holographical fabricated MDG showed DE of $96 \%$ at $1053 \mathrm{~nm}$ in the first order for TE polarization, as shown in Fig. 9. The experimental results were in good agreement with the calculation results. ${ }^{112} \mathrm{Li}$ et al. pointed out that multilayer dielectric (MLD) stack showed high reflectivity, low absorption, and high LIDT. The theoretical results indicated that the peak DE was nearly to $100 \%$. They directly deposited nine layers, $(\mathrm{HL})^{4} \mathrm{H}$, of $\mathrm{ZnS} / \mathrm{Na}_{3} \mathrm{AlF}_{6}$ on the etched photoresist gratings. The test results showed that the DE was $70 \%$ and $84 \%$ corresponding to incidence from air and substrate, respectively. They inferred that the discrepancy between the theory and the experiment resulted from the nonconformally coated gratings, which was unavoidable for directly deposited grating on photoresist. The key for further improving DE was improving the conformability of the coated dielectric layers. Finally, they pointed out that three considerations answered for the situation all dielectric gratings have not been developed. (i) The coated multilayer gratings were not suitable 


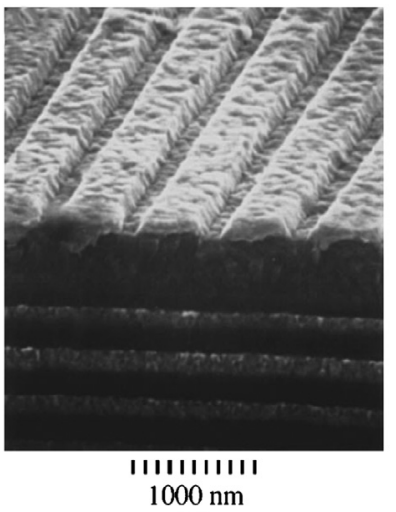

(a)

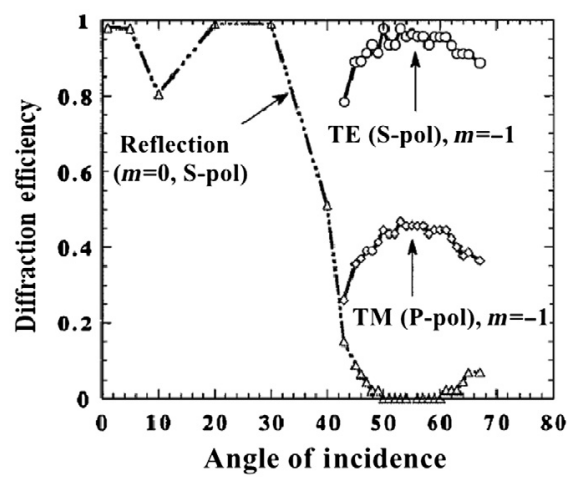

(b)

Fig. 9 The property of fabricated MDG: (a) SEM image of the MDG and (b) the DE of MDG in (a). The first-order TM polarization: solid curve, open squares; the first-order TE polarization: solid curve, open circles; and zeroth reflection TE polarization: dotted-dashed curve, open triangles. The incident wavelength was $1053 \mathrm{~nm} .{ }^{112}$

for spectroscopic applications due to the existence of a large number of diffraction anomalies. (ii) There were no tools for designing coated multilayer gratings. (iii) It was difficult to deposit conformable thin film coatings. ${ }^{113}$ In 1996, Britten et al. first proposed that alternating quarterwave layers of $\mathrm{HfO}_{2}$ and half-wave layers of $\mathrm{SiO}_{2}$ (HLL) can be utilized to fabricate MLD mirror, and the surface relief can be etched into the top layer either $\mathrm{HfO}_{2}$ or $\mathrm{SiO}_{2}$. The calculation predicted that obtaining the same $\mathrm{DE}$, the theoretical groove depth of $\mathrm{SiO}_{2}$ was deeper $300 \mathrm{~nm}$ than that of $\mathrm{HfO}_{2}$, which was in good agreement with the experiments, as shown in Fig. 10. Figure 10(a) shows the MDG etched on $\mathrm{HfO}_{2}$ top layer. Its DE was $95 \%$ for TE polarization at $1053 \mathrm{~nm}$, which was lower than the designed $99 \%$. This may be attributed to the thinner thickness than the designed $294 \mathrm{~nm}$. The LIDT was $0.21 \mathrm{~J} / \mathrm{cm}^{2}$ at $1053 \mathrm{~nm}$ for $300 \mathrm{fs}$ pulse. The LIDT was disappointing and lower than the gold-overcoated photoresist gratings. They claimed that it may be influenced by the preferential loss of oxygen in the etching process, resulting in reduced LIDT. Figure 10(b) shows the MDG etched on $\mathrm{SiO}_{2}$ top layer with DE of $94 \%$. The LIDT was $0.51 \mathrm{~J} / \mathrm{cm}^{2}$, which was about $25 \%$ higher than that of gold-overcoated photoresist gratings. It was encouraging for the improvement of LIDT. However, more investigations should be executed to further improve the LIDT of MDGs. Meanwhile, because of its good optical property and high laser resistance, $\mathrm{HfO}_{2} / \mathrm{SiO}_{2}$ has been the main stream materials in MDGs. ${ }^{100,101}$

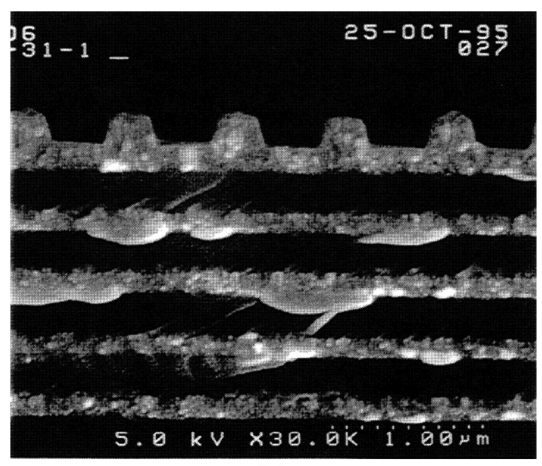

(a)

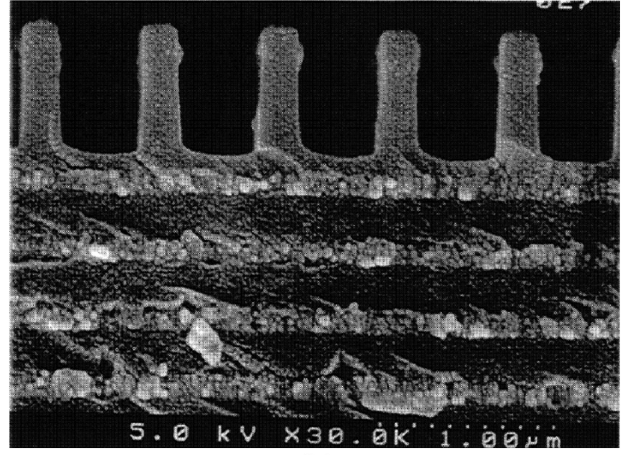

(b)

Fig. 10 SEM images of fabricated MDGs. (a) SEM of HLL MDG etched into $\mathrm{HfO}_{2}$ top layer with DE of $95 \%$ for TE polarization at $1053 \mathrm{~nm}$. Its groove depth was $200 \mathrm{~nm}$ and duty cycle was 0.4 . (b) SEM of HLL MDG etched into $\mathrm{SiO}_{2}$ top layer with DE of $94 \%$ for TE polarization at $1053 \mathrm{~nm}^{114,115}$ 


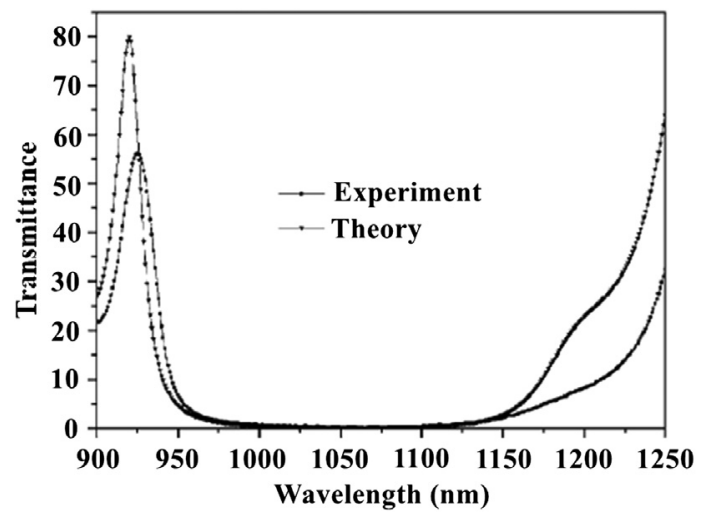

Fig. 11 The comparison of optical properties between theory and experiment. The stack was H3L(H2L)^9H0.5L2.03H. ${ }^{118,119}$

In 1999, Hehl et al. confirmed that the theoretical DE of nearly $100 \%$ can be obtained. The MLD mirror was deposited with $\mathrm{Nb}_{2} \mathrm{O}_{5}\left(n_{\mathrm{H}}=2.375\right)$ and $\mathrm{SiO}_{2}\left(n_{\mathrm{L}}=1.46\right)$ on a plane fusedsilica substrate. The grating was etched into the top layer of $\mathrm{SiO}_{2}$ by ion beam etching. Its $\mathrm{DE}$ was $97 \%$ in the first order for TE polarization at $532 \mathrm{~nm}$. The LIDTs were 4.4 and $0.18 \mathrm{~J} / \mathrm{cm}^{2}$ under pulse duration of $5 \mathrm{~ns}$ and 1 ps at $532 \mathrm{~nm} .{ }^{116}$ In 2003, Wei et al. investigated the basic principle for the generation of high DE of MDG utilizing $S$ matrix method and identified the conditions for achieving high DE. They concluded that the diffraction of the grating can be explained as the interference of a symmetric wave and an antisymmetric wave. The high DE can be achieved when the two left diffracted waves generated by the symmetric and antisymmetric incident waves were in phase. The analysis provided good guidance for the design of high-efficiency MDGs. ${ }^{117}$

In 2005, Kong et al. designed and analyzed a MLD mirror used in PCGs, the stack of $\mathrm{H} 3 \mathrm{~L}(\mathrm{H} 2 \mathrm{~L})^{\wedge} 9 \mathrm{H} 0.5 \mathrm{~L} 2.03 \mathrm{H}$, where $\mathrm{H}$ is $\mathrm{HfO}_{2}$ and $\mathrm{L}$ is $\mathrm{SiO}_{2}$. The transmittance was $0.29 \%$ at $1053 \mathrm{~nm}$ with $51.2 \mathrm{deg}$ for TE polarization. The bandwidth was $70 \mathrm{~nm}$ centered at $1053 \mathrm{~nm}$ with the reflectivity $>99.5 \%$. The experimental transmission was well agreement with the theoretical design, as shown in Figs. 11. ${ }^{118,119}$

Non-uniform optical near-field distribution was one of the important factors limiting the LIDT of MDGs. Consequently, optimizing the electric field distribution in MDGs was an important means to further improve the LIDT. In 2006, Liu et al. deeply investigated the electric field distributions in gratings and multilayer film region using Fourier modal method. The near-field distributions in the gratings ridge was closely related to the gratings parameters, such as top layer thickness, groove depth, duty cycle, and gratings material. They developed a merit function to balance the DE and the electric field enhancement in the gratings ridge. After optimization, the grating parameters can be obtained with lowest electric field enhancement, thus, higher LIDT, as shown in Fig. 12. ${ }^{120-122}$ Néauport et al. fabricated several samples with different electric field distributions inside the MDGs. The close optical and AFM inspection of damage sites showed that damage occurs where the electric field was maximum calculated using the differential method. Figure 13 shows the electric field distributions of two MDGs with different parameters. Figure 13(a) shows obvious electric field enhancement in grating ridge and interface compared to that of Fig. 13(b). The test showed that the LIDTs were 3.6 and $4.5 \mathrm{~J} / \mathrm{cm}^{2}$ for MDGs of (a) and (b), respectively. It was verified that the LIDT of MDGs was determined by the value of $E^{2} .{ }^{123}$

In 2010, Wang et al. analyzed the restriction factors of widening bandwidth of MDGs, including the reflectivity bandwidth of MLD mirror and the guided-mode resonance (GMR) phenomenon. The existence of GMR in MDG would destroy the pulse spectrum shape and depress the LIDT of MDG. They proved that the bandwidth of MDG was determined by the bandwidth of high-reflectivity mirror for the first order transmitted diffraction. They inferred that reducing grating period was an effective approach to eliminate GMR in MDG, thus, broadening the bandwidth of MDG. ${ }^{124}$ Later, they designed an MDG with the groove depth $<80 \mathrm{~nm}$ using particle swarm optimization algorithm and Fourier modal method, which was much shallower 


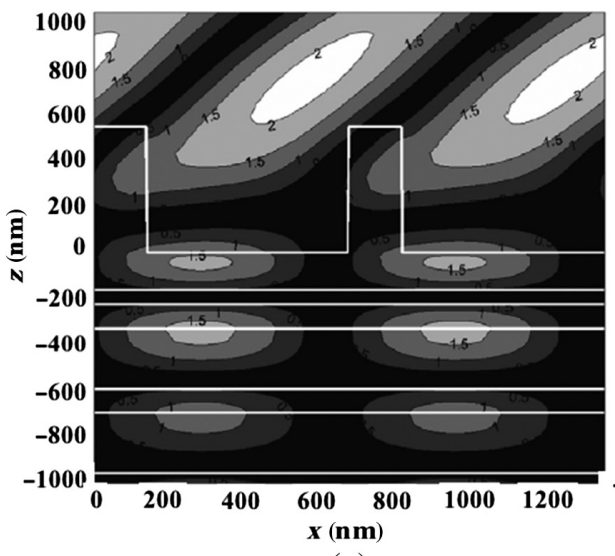

(a)

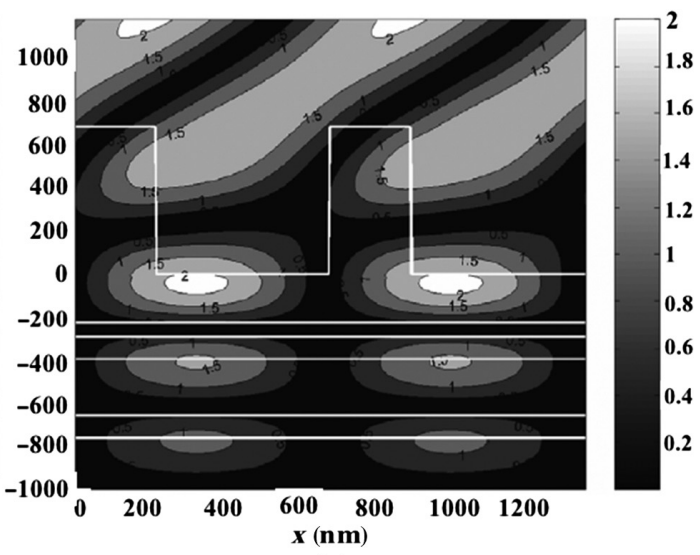

(b)

Fig. 12 Near-field distributions of MDGs after optimization: (a) $\mathrm{HfO}_{2}$ top layer and (b) $\mathrm{SiO}_{2}$ top layer. The top layer thickness, groove depth, and duty cycle were $700 \mathrm{~nm}, 540 \mathrm{~nm}$, and 0.22 in (a) and $910 \mathrm{~nm}, 684 \mathrm{~nm}$, and 0.32 in (b), respectively. ${ }^{120}$

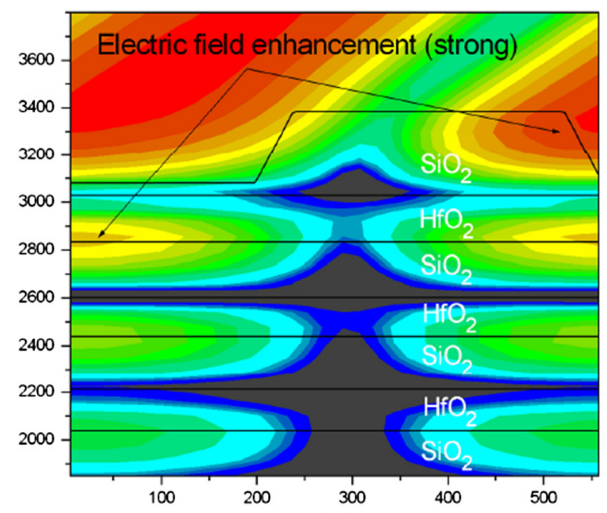

(a)

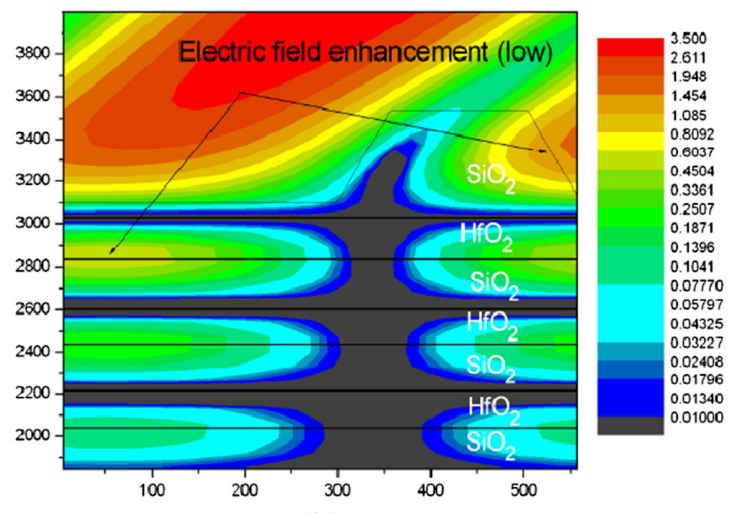

(b)

Fig. 13 The electric field distributions in MDGs with different parameters. The residual layer thickness, groove depth, and duty cycle were $0 \mathrm{~nm}, 303 \mathrm{~nm}$, and $0.53 \mathrm{in}$ (a) and $18 \mathrm{~nm}, 440 \mathrm{~nm}$, and 0.35 in (b), respectively. ${ }^{123}$

than the reported MDGs. The shallow groove depth was beneficial for the grating etch process. Its bandwidth was $60 \mathrm{~nm}$ with DE higher than $97.5 \%$ centered at $800 \mathrm{~nm} .^{125,126}$

The DE and bandwidth of MDGs have been enough optimized. ${ }^{127-138}$ Afterward, the investigations mainly focused on the improvement of LIDT, which can be achieved through optimizing the structure and the fabrication technology, which will be discussed in Sec. 4.2.3.

\subsubsection{Metal multilayer dielectric gratings}

The MDG often consisted of several pairs of alternating high and low dielectric film, which would cause mechanical stress among the multilayer stack. The mechanical stress can lead to craze and reduce its properties, as shown in Fig. $14 .{ }^{139}$ For this reason, the MMDG model was first proposed in 2006 by Bonod and Néauport ${ }^{140}$ One layer metal was inserted between the substrate and the MLD stack, and some pairs of dielectric bilayers were replaced. The number of bilayers was decreased, and thus the mechanical stress was reduced within the stack, which effectively improves the DE, LIDT, and the stability of MMDG. Theoretical calculation showed that the number of bilayers can be decreased to 7 from 9. This was the first report and prototype of MMDG. Afterward, this research group devoted themselves to the investigation of MMDGs and effectively improved the optical performance of MMDGs. ${ }^{140-142}$ 


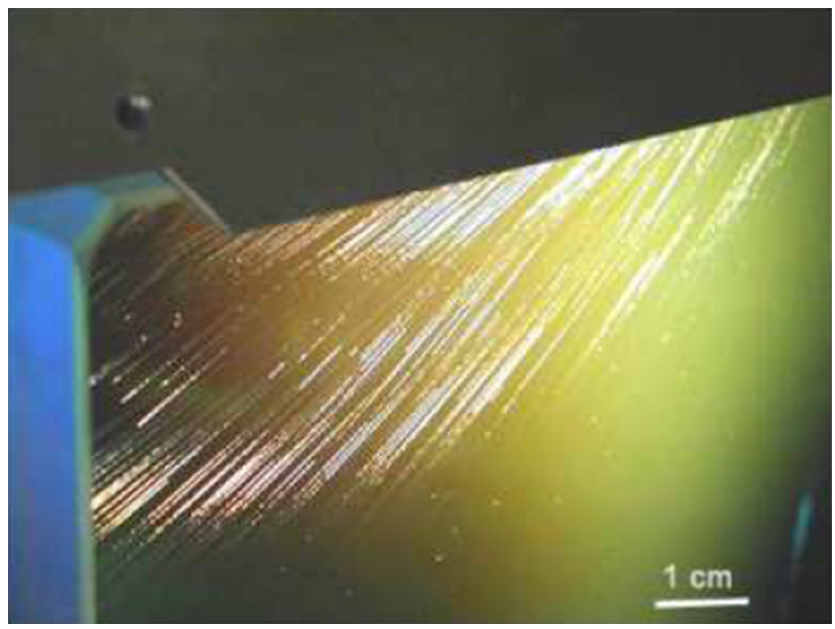

Fig. 14 Crazing phenomenon observed on an $\mathrm{HfO}_{2} / \mathrm{SiO}_{2}$ high-reflection e-beam evaporated multilayer dielectric mirror. It was result from the mechanical stress among the multilayer stack. ${ }^{139}$

With the development of MMDGs, it was found that the insertion of metal layer can not only decrease the mechanical stress, but also broaden the bandwidth. When the pulse duration was further compressed to femtoseconds, the bandwidth of pulse can reach to $100 \mathrm{~nm}$ and even $200 \mathrm{~nm}$. The work bandwidth of MDG cannot meet the performance requirements. ${ }^{143,144}$ Since metal layer always showed good reflectivity over broad bandwidth, Flury et al. reported a high-efficiency wide-band MMDG. It showed DE higher than 95\% over $200 \mathrm{~nm}$ wavelength range centered at $800 \mathrm{~nm}$ for TE polarization. The experimental result verified that the DE of the first order can reach to $98 \%$ and was coincide well with theoretical simulation, as shown in Fig. 15. This kind of MMDG was a potential to realize high-efficiency CPA of femtosecond pulses as short as $20 \mathrm{fs}$. However, they did not test the LIDT of the MMDG. ${ }^{145}$

Kong et al. have committed to the work of novel structure design, performance analysis, and optimization. First, Ag layer was inserted between substrate and bilayers, the MMDG showed DE higher than $97 \%$ over $130 \mathrm{~nm}$ bandwidth centered at $800 \mathrm{~nm}$, and DE higher than $97 \%$ over $154 \mathrm{~nm}$ bandwidth centered at $1053 \mathrm{~nm}$ for TE polarization, respectively. ${ }^{146}$ Later, with three kinds of dielectric materials, $\mathrm{HfO}_{2}, \mathrm{SiO}_{2}$, and $\mathrm{Ti}_{2} \mathrm{O}_{5}$, the designed MMDG showed DE higher than $97 \%$ over $150 \mathrm{~nm}$ bandwidth centered at $800 \mathrm{~nm},{ }^{147}$ DE higher than $97 \%$ over $195 \mathrm{~nm}$ bandwidth centered at $1053 \mathrm{~nm}$ for TE polarization, respectively. ${ }^{148}$ To further decrease the number of dielectric layers, MMDGs, consisted of only single pair of bilayers, were designed. And they showed DE higher than $97 \%$ over $120 \mathrm{~nm}$ bandwidth centered at $800 \mathrm{~nm},{ }^{149}$ and DE higher than $97 \%$ over $160 \mathrm{~nm}$ bandwidth centered at $1053 \mathrm{~nm}$ for TE polarization,

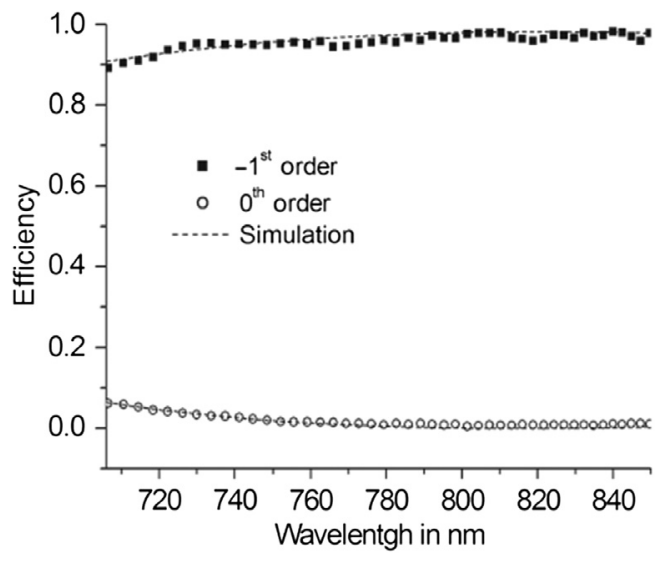

Fig. 15 The experimental first-order DE and zeroth reflected order spectra under incidence of 50 deg for TE polarization. ${ }^{145}$ 


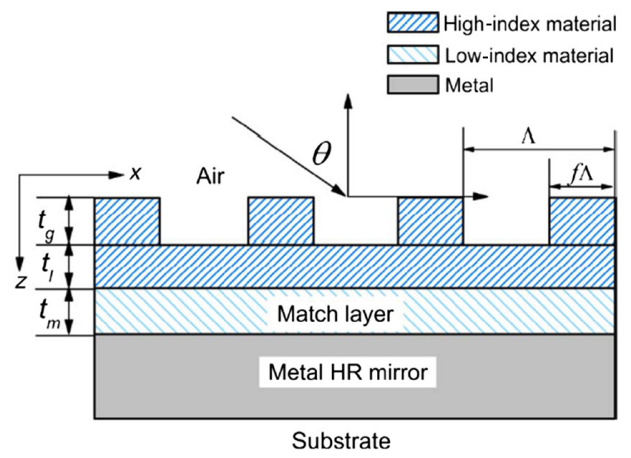

(a)

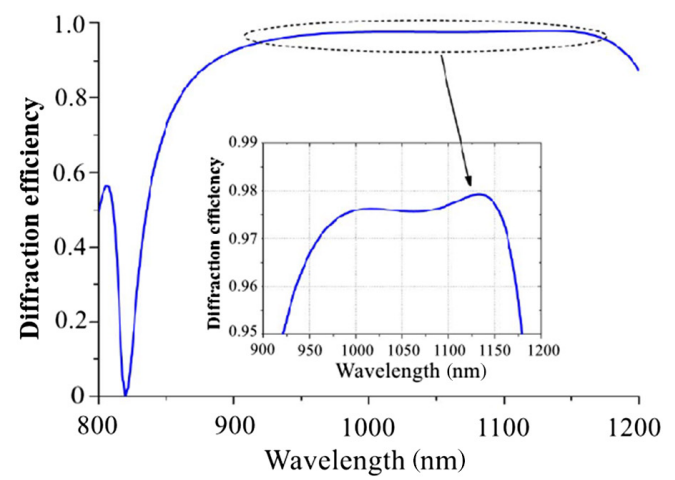

(b)

Fig. 16 (a) Structure of a new MMDG with one metal layer, one low-index dielectric layer, and one high-index dielectric layer with etched groove depth. (b) Ultrabroad top-hat DE spectrum of (a). Its parameters were: groove depth, $315 \mathrm{~nm}$; residual thickness, $173 \mathrm{~nm}$; low-index layer thickness, $140 \mathrm{~nm}$; and duty cycle, $0.25 .^{153}$

respectively. ${ }^{150}$ The above MMDGs exhibited good optical performance and favorable fabrication tolerance. It provided good theoretical guidance for the fabrication of MMDGs. However, they did not show the corresponding fabrication technology and product. ${ }^{151,152}$

In 2010, Wang et al. reported a new MMDG, consisted of $\mathrm{Ag}, \mathrm{HfO}_{2}$, and $\mathrm{SiO}_{2}$, with $\mathrm{DE}$ higher than $97 \%$ over $200-\mathrm{nm}$ bandwidth centered at $1053 \mathrm{~nm}$ for TE polarization. It only consisted of a metal layer, a low-index material layer, and a high-index material with etched groove depth, as shown in Fig. 16. The number of total layers was minimized, which was beneficial for the fabrication. ${ }^{153}$ In 2013, Guan et al. designed an MMDGs centered at $800 \mathrm{~nm}$. The DE, bandwidth, and near-field distributions were theoretically analyzed in detail, especially the effect of single match layer and multimatch layers on the optical performance of MMDGs. The MMDG with single match layer showed that the minimum electric field distributes in the metal layer, and the maximum electric field distributes in grating ridge, which can improve the LIDT of MMDG. If the thickness and refractive index of the match layer were changed, the maximum electric field in the grating ridge, match layer, and metal layer increased with the decrease of DE. For the MMDG with multidielectric match layers, the bandwidth and the maximum electric field in the metal layer decreased with the increase of bilayers, and the maximum electric field in the grating ridge and match layer decrease, as shown in Fig. 17. The maximum bandwidth and minimum electric field should be balanced according to the requirement. ${ }^{154,155}$

The previous gratings were all designed aiming at sole either TE or TM polarization. In 2012, $\mathrm{Hu}$ et al. designed a polarization-independent wideband MMDG. The MMDG consisted of a metal layer and a connecting layer, and the rectangular groove was etched on the top dielectric layer. The MMDG exhibited the DE higher than $90 \%$ over $120 \mathrm{~nm}$ bandwidth centered at $800 \mathrm{~nm}$ for both TE and TM polarization, as shown in Fig. 18. This MMDG had good potential applications in laser systems and spectrometers. ${ }^{156}$

\subsection{Fabrication Technologies of PCGs}

The final aim of PCGs was the application in laser systems. And the fabrication of PCGs was much critical to the final optical performance. Consequently, the fabrication technologies of PCGs should draw more attentions.

\subsubsection{Metal gratings}

The systematical investigation of ACGs has suspended for several years imputing its difficult improvement of LIDT. ${ }^{157,158}$ However, until now, ACG was difficult to be absolutely replaced for femtosecond pulses (especially for pulse shorter than $100 \mathrm{fs}$ ) attributed to its broad bandwidth. ${ }^{159,160}$ In 2013, Poole et al. found that the LIDT has no dependence on laser pulse duration for femtosecond laser, but showed clear dependence on Au surface morphology. 


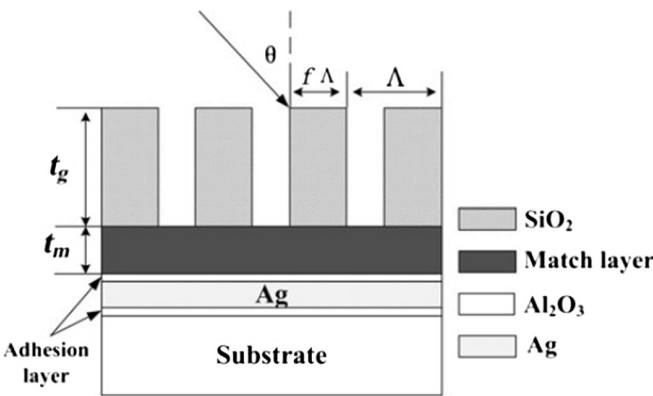

(a)

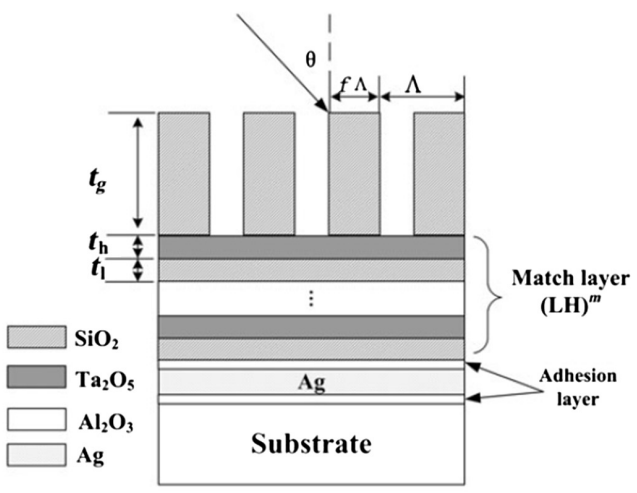

(c)

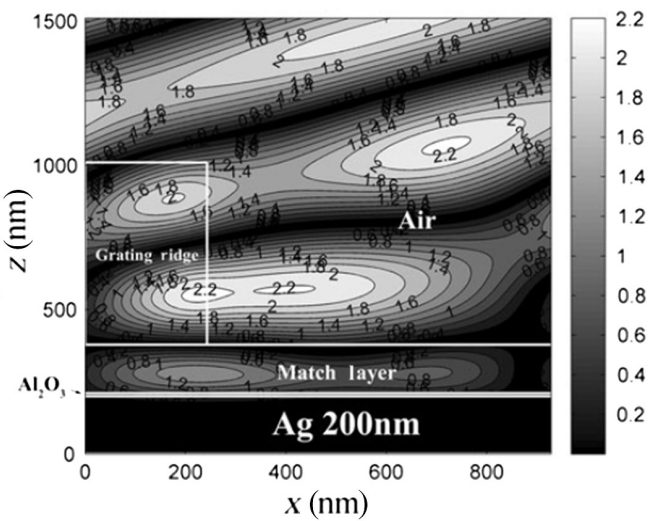

(b)

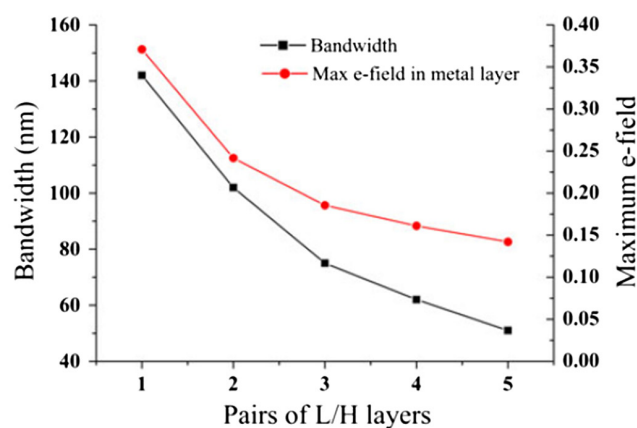

(d)

Fig. 17 (a) Structure of MMDG with single dielectric match layer. (b) The electric field distribution with parameters of MMDG: groove depth, $632 \mathrm{~nm}$; match layer thickness, $169 \mathrm{~nm}$; period, $931 \mathrm{~nm}$; and duty cycle, 0.26 . (c) Structure of MMDG with multidielectric match layers. (d) The changes of bandwidth and maximum electric field in the Ag layer versus the number of alternating bilayers. ${ }^{154}$

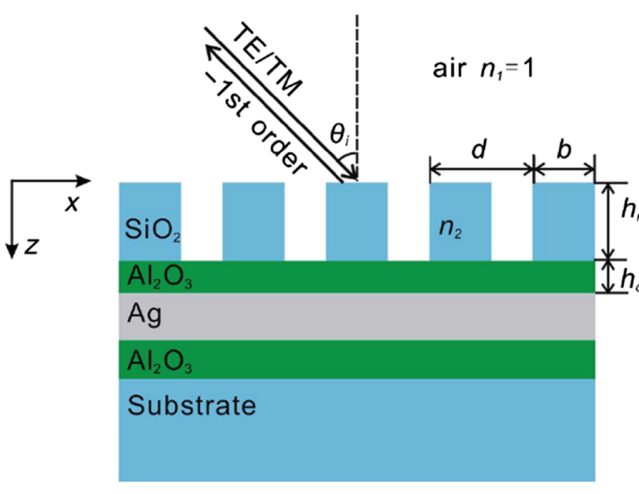

(a)

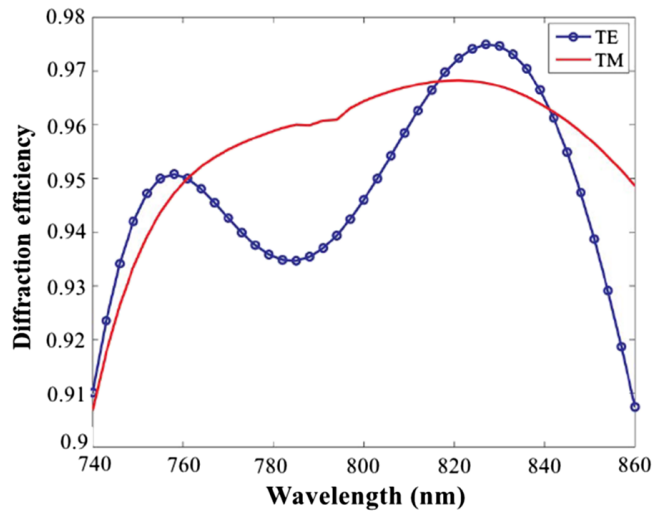

(b)

Fig. 18 (a) Schematic of the MMDG: $n_{1}$ and $n_{2}$ are refractive indices of grating grooves and ridges, respectively; $\theta_{i}$, incident angle; $d$, grating period; $b$, ridge width; $h_{r}$, grating depth; and $h_{c}$, thickness of connecting layer. (b) The first-order diffraction efficiencies for both TE and TM polarizations. ${ }^{156}$

Figure 19 shows the SEM images of the grating with different coating techniques. Electromagnetic field modeling showed that non-conformal (NC) coating morphology aroused significant local field enhancement near groove edges, lowering the DE and LIDT. And the experimental results verified the effectiveness of the model. As shown in Fig. 20, it was found that the conformal coating showed obvious higher LIDT than NC coating. Meanwhile, the LIDT 


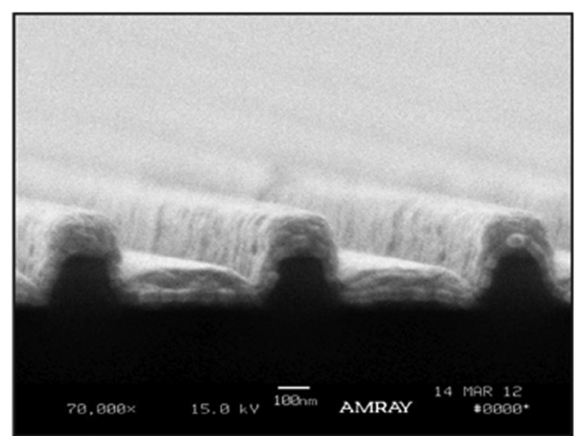

(a)

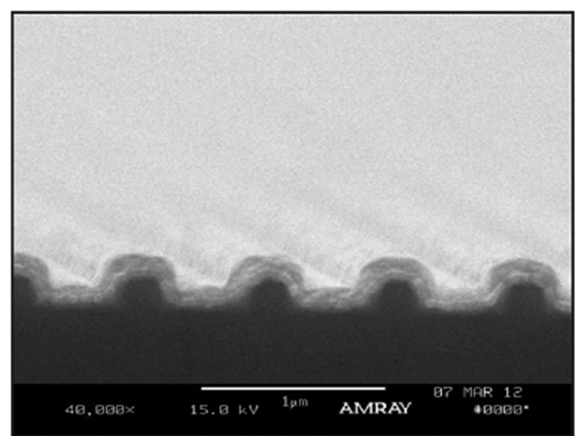

(b)

Fig. 19 SEM image of fabricated gratings: (a) conventional sputter-coated grating showing an NC groove structure and (b) energetic sputter-coated grating showing more uniform groove structure. ${ }^{106}$

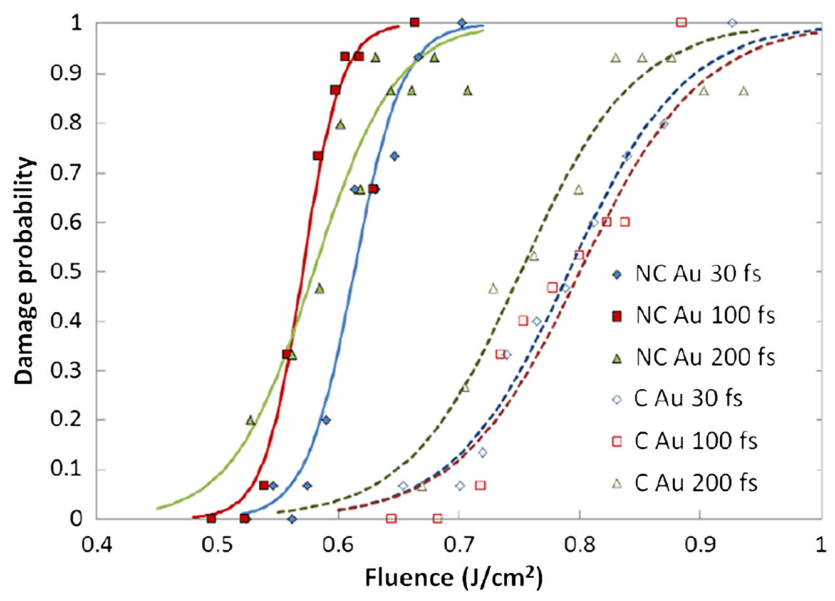

Fig. 20 The dependence of pulse duration on LIDT. While the conformal $(C)$ coating showed higher LIDT than the NC coating, and there was no clear pulse duration dependence of the LIDT. $^{106}$

had no dependence on pulse duration. Finally, they testified that the Ag-coated grating performed higher LIDT than ACG, which maybe a satisfactory substitute. ${ }^{106}$ In 2014, Li et al. theoretically investigated the performance of ACGs with different profiles, such as rectangular, sinusoid, and semisinusoid using RCWA. ${ }^{161}$ It was found that the duty cycle should be excess of 0.5 for the rectangular or semisinusoidal groove, and duty cycle would cause DE to change sharply at the short wavelength. The ACG fabricated with holographic recording method exhibited DE excess of $94 \%$ for TM polarization at $808 \mathrm{~nm}$, as shown in Fig. 21. In 2015, Muhutujiang et al. investigated an approach, in which the gratings patterns were generated by directly etching the quartz substrate. Its fabrication process was as follows: (i) preparation of a 220-nm-thick photoresist layer on a quartz substrate; (ii) holographic exposure on the photoresist layer; (iii) developing the grating masks; (iv) ion beam etching the quartz substrate; and (v) depositing gold on the substrate grating. The fabricated ACG showed good DE, the average DE was $89.2 \%$ over the wavelength 750 to $850 \mathrm{~nm}$ for TM polarization, and the peak DE is $90 \%$. The SEM image of the etched grating is shown in Fig. $22 .{ }^{162}$ Recently, Jin et al. have deeply studied the damage process and the effect on LIDT for different fabrication methods, such as magnetron sputtering and e-beam evaporation. The LIDTs were 0.59 and $0.43 \mathrm{~J} / \mathrm{cm}^{2}$ for ACGs fabricated by magnetron sputtering and e-beam evaporation at pulse duration of $60 \mathrm{fs}$, respectively. It showed that the adhesion between the gold film and the photoresist determined the damage behavior, and the magnetron sputtering can produce ACGs with better adhesion, thus, improving the LIDT. $^{94,163}$ 


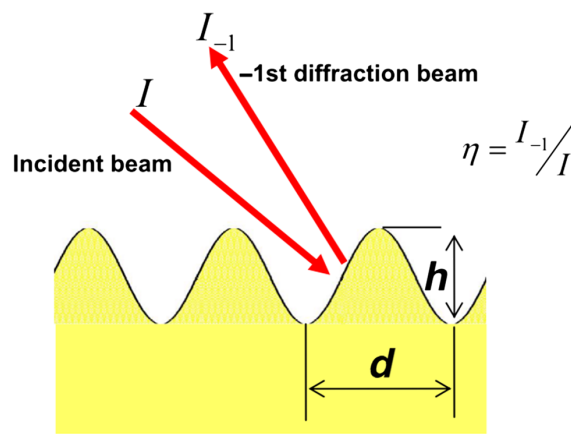

(a)

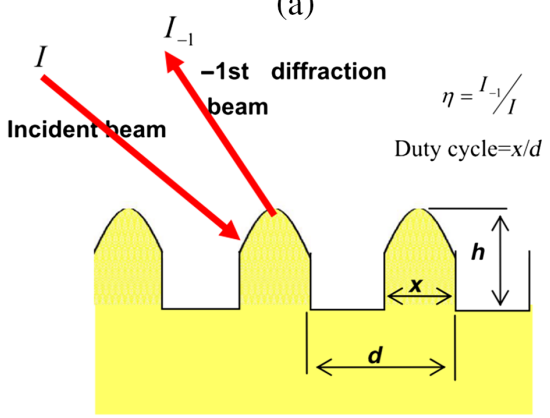

(c)

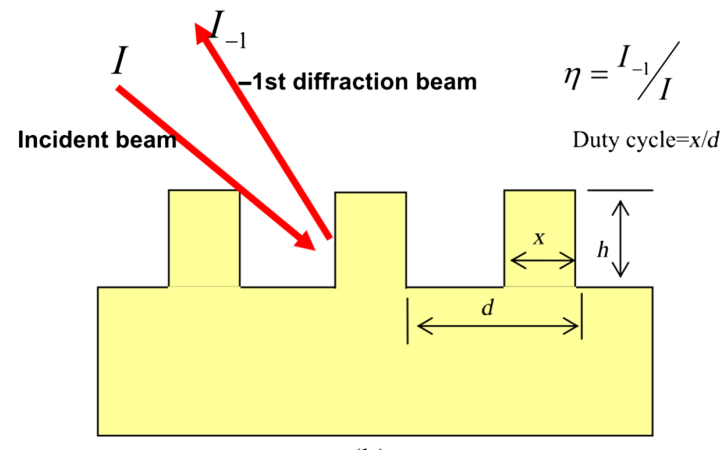

(b)

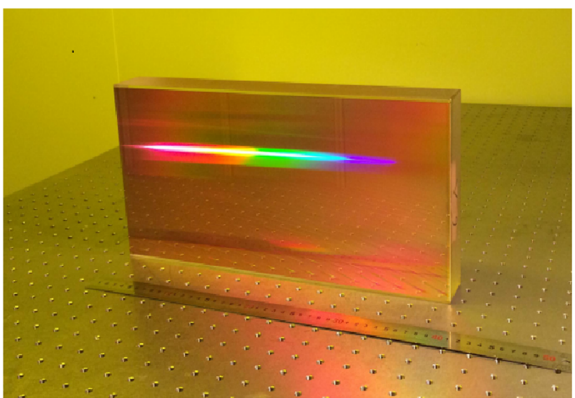

(d)

Fig. 21 ACGs with different profiles: (a) sinusoid; (b) rectangular; (c) semisinusoid; and (d) the fabricated grating with aperture of $200 \mathrm{~mm} \times 400 \mathrm{~mm} .^{161}$

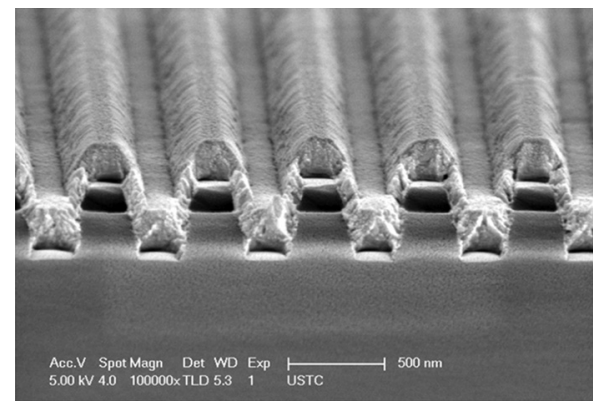

Fig. 22 SEM image of fabricated grating. The sinusoid profile ACG with line densities of $1740 \mathrm{l} /$ $\mathrm{mm}$. Au layer thickness was $200 \mathrm{~nm} .^{162}$

\subsubsection{Multilayer dielectric gratings}

Previous work has adequately reported the theoretical design and experimental results on DE and LIDT. However, fabrication technology, which was a much important procedure for the realization of the designed properties, has been barely reported. In 2005, Oliver et al. focused on the fabrication of an MLD to allow the fabrication of a grating with higher DE and LIDT and investigated the effect of MLD on the holographic exposure quality. By modifying the MLD structure and suppressing the reflectance of the MLD coating during holographic exposure, the straighter sidewalls of the grating pillars can be obtained, thus, yielding a higher quality MDG with greater control of duty cycle. ${ }^{164}$

In 2006, Ashe et al. systematically investigated the effectiveness of some wet-chemical cleaning processes on gratings for improving the DE and LIDT, which were commonly used in semiconductor chemical cleaning processes. It was found that the DE and LIDT can together be improved with the Piranha cleaning process. The SEM images showed no visual contamination after cleaning. This was promising for improving the optical performance of MDGs. ${ }^{165,166}$ In 2006, Kong et al. studied the effect on LIDT of MDGs for different fabrication processes. 


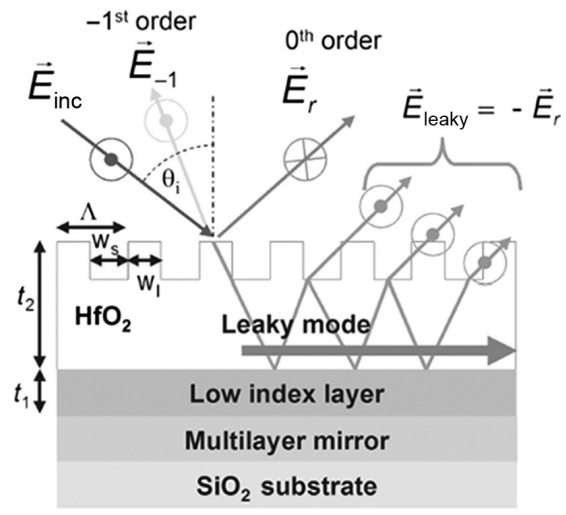

(a)

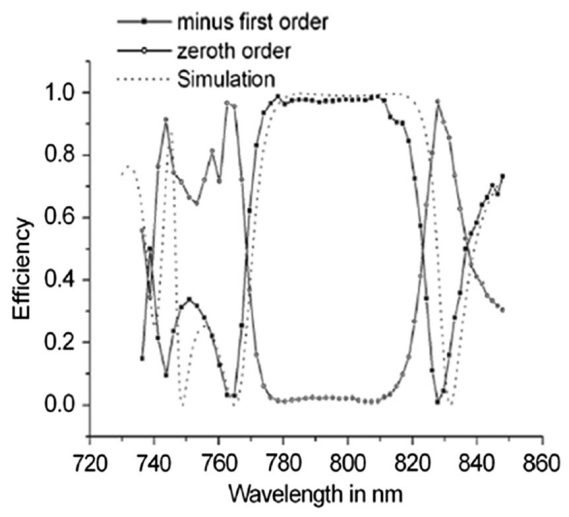

(b)

Fig. 23 (a) Cross-sectional view of the dielectric mirror-based leaky mode propagating in the high-index layer and (b) experimental DE spectrum. The first-order average DE was $97 \%$ over 777 to $815 \mathrm{~nm}$ under an incidence angle of $57 \mathrm{deg}$ for TE polarization. ${ }^{170}$

They found that the LIDT of MDG was obviously lower than that of MLD and the cleaning of grating was beneficial for improving LIDT. Finally, they pointed out that the following methods may improve the LIDT of MDGs. (i) Modifying the electric field distributions makes the peak electric field not to distribute in the unsubstantial district. (ii) Modifying the fabrication technology decreases the defects and impurity in the films. (iii) The MLD can be cleaned by surface treatment technology. (iv) Modifying the technology of e-beam etching reduces the change of $\mathrm{HfO}_{2}$ stoichiometric proportion. ${ }^{167-169}$

In 2007, Lyndin et al. designed and fabricated an all-dielectric grating with top-hat high DE over a broad spectral. As shown in Fig. 23(a), the grating was etched in high-refractive index layer instead of the traditional low-index silica layer. The experimental result indicated that the groove depth was $123 \mathrm{~nm}$ and duty cycle was 0.38 . The average DE is $97 \%$ over 777 to $815 \mathrm{~nm}$ under an incidence angle of $57 \mathrm{deg}$ for TE polarization, which was well agreement with the theoretical simulation, as shown in Fig. 23(b). The zeroth order DE was $2 \%$ on the average and exhibited sharp peaks at the band edges. ${ }^{170}$

In 2007, Liu et al. proposed that the $\mathrm{SiO}_{2}$ material as the top layer of the MLD mirror for grating fabrication was beneficial for improving the LIDT, and it was experimentally verified. They designed an optimal design of (HLL)^9H as the MLD, the $\mathrm{SiO}_{2}$ top layer was optimized considering the DE and the electric field enhancement. The average LIDT was $10 \mathrm{~J} / \mathrm{cm}^{2}$ under $12 \mathrm{~ns}$ pulses at $1053 \mathrm{~nm} .^{171,172}$

Liu et al. specially investigated the effect of the photoresist gratings with different profiles as mask on the transferred grating profiles. They concluded that it was necessary to utilize high and steep enough photoresist grating mask to obtain grating with vertical profiles, as shown in Fig. 24. ${ }^{173,174}$

Recently, the investigations for the effect of electric field on LIDT were attractive. These works focused on optimization of the electric field distributions in the grating ridges and the damage process, the LIDT can be improved. ${ }^{175-185}$ Meanwhile, much research also verified that the LIDT can also be improved through cleaning the surface, which can effectively reduce the defects and impurity. ${ }^{186-189}$ Howard et al. proposed a chemical cleaning process to eliminate the contaminants. Figure 25 shows that BARC and photoresist layers on the pillar tops were effectively removed and the grating pillars were narrowed after the cleaning process. ${ }^{192}$

\subsubsection{Metal multilayer dielectric gratings}

In 2007, Canova et al. fabricated an MMDG with a silver mirror, a 30-nm protective layer of $\mathrm{Al}_{2} \mathrm{O}_{3}$ and the grating etched in $\mathrm{HfO}_{2}$ layer, as shown in Fig. 26(a). It exhibited a 140-nm broad bandwidth with the average DE of 95\%, as shown in Fig. 26(b). They investigated the fabrication technology of MMDG and pointed out that there were the following difficulties in the fabrication process of MMDGs. (i) There was no adhesion layer between metal and dielectric to bond them 


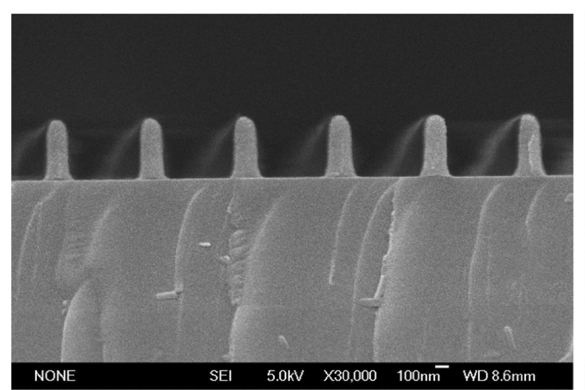

(a)

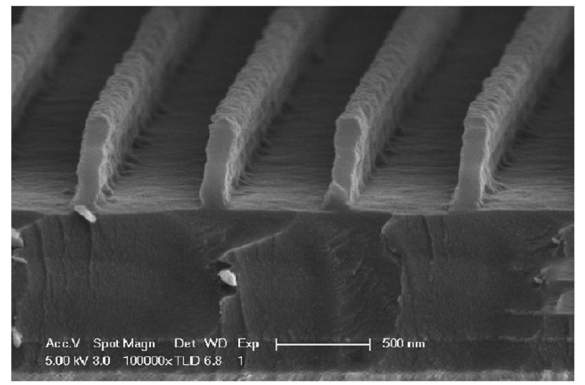

(c)

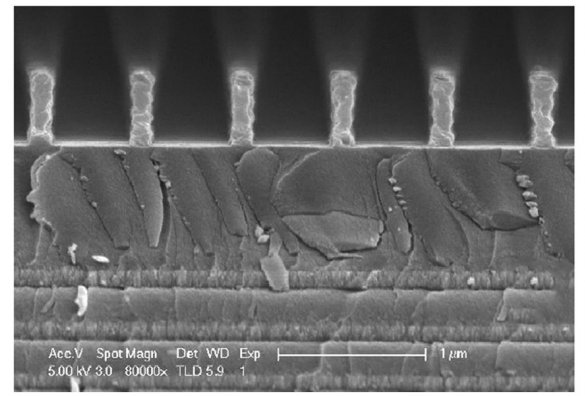

(e)

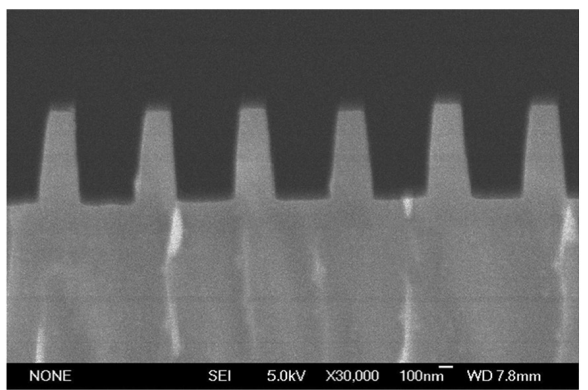

(b)

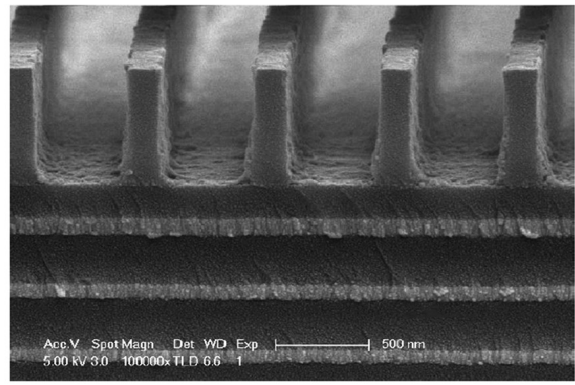

(d)

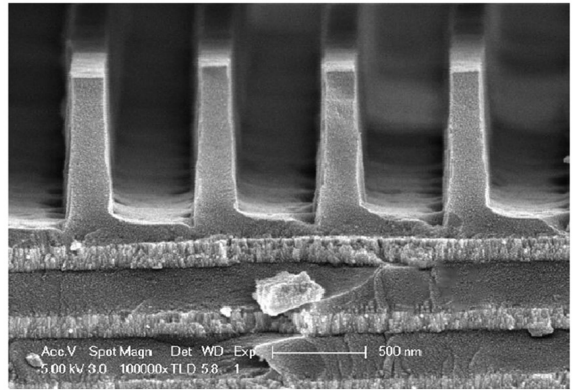

(f)

Fig. $24 \mathrm{SEM}$ images of photoresist grating and the etched $\mathrm{SiO}_{2}$ grating with different profile masks. (a) Original photoresist grating mask I: groove depth $\sim 420 \mathrm{~nm}$, duty cycle $\sim 0.22$, and sidewall angle $\sim 85$ deg. (b) Grating etched into $\mathrm{SiO}_{2}$ with mask I: groove depth $\sim 485 \mathrm{~nm}$, duty cycle $\sim 0.36$, sidewall angle $\sim 85$ deg. (c) Original photoresist grating mask II: groove depth $\sim 540 \mathrm{~nm}$, duty cycle $\sim 0.19$, and sidewall angle $\sim 90$ deg. (d) Grating etched into $\mathrm{SiO}_{2}$ with mask II: groove depth $\sim 690 \mathrm{~nm}$, duty cycle $\sim 0.23$, and sidewall angle $\sim 90$ deg. (e) Original photoresist grating mask III: groove depth $\sim 550 \mathrm{~nm}$, duty cycle $\sim 0.19$, and sidewall angle $\sim 90$ deg. (f) Grating etched into $\mathrm{SiO}_{2}$ with mask II: groove depth $\sim 960 \mathrm{~nm}$, duty cycle $\sim 0.22$, and sidewall angle $\sim 90$ deg. ${ }^{171}$

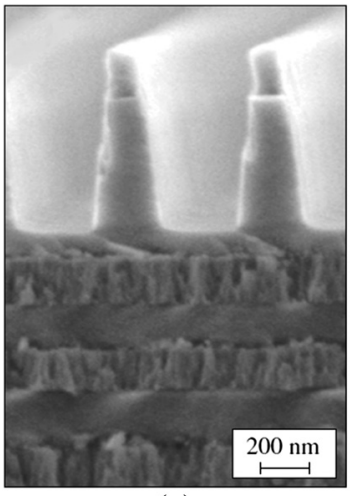

(a)

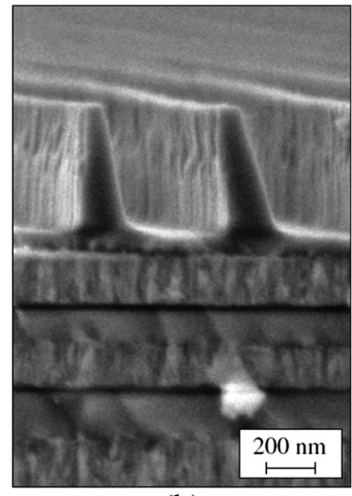

(b)

Fig. 25 SEM images showing MDG cross section (a) before chemical cleaning and (b) after cleaning. The cleaning process removed BARC and photoresist layers form the pillar tops and narrowed the grating pillars. ${ }^{189}$ 


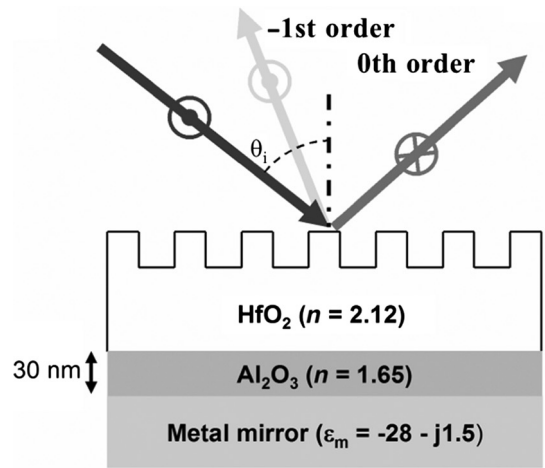

(a)

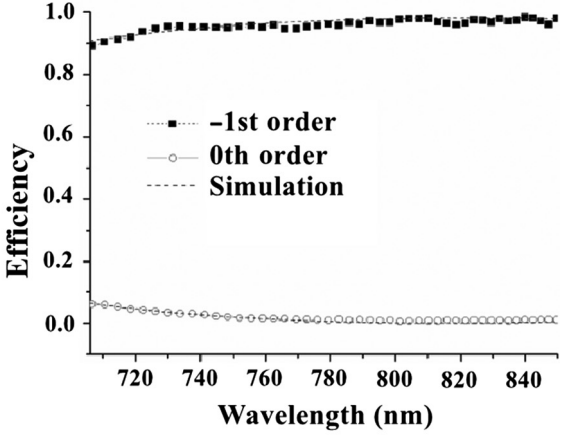

(b)

Fig. 26 (a) Cross-sectional view of the MMDG and (b) the experimental and theoretical first-order DE and zeroth reflected spectra under incidence of 57 deg for TE polarization. ${ }^{190}$

and avoid delamination. (ii) The dielectric layers cannot be handled by high-temperature annealing for removing possible defects, which may decrease the LIDT. (iii) It was difficult to expose interferogram on the materials before the high-refractive index materials. (iv) To assure the physically and chemically intactness of the metal mirror surface in the process of grating etching, the etching chemistry and sputtering conditions were severely restricted. Although there were too much technical difficulties and influences, the LIDT of MMDG was comparative with that of MDG, $1.1 \mathrm{~J} / \mathrm{cm}^{2}$, which verified that the introduction of metal layer has no influence on the LIDT. ${ }^{190}$

In 2009, Palmier et al. experimentally compared the reflectivity and LIDT properties of metal multilayer dielectric (MMLD) mirror (Pyrex/20 nm Cr $150 \mathrm{~nm} \mathrm{Au}\left(246 \mathrm{~nm} \mathrm{SiO} 2155 \mathrm{~nm} \mathrm{HfO} \mathrm{H}_{2}\right.$ ) ^4) $579 \mathrm{~nm} \mathrm{SiO}_{2}$ and MLD mirror $\left(\operatorname{Pyrex} /\left(115 \mathrm{~nm} \mathrm{HfO}_{2} 311 \mathrm{~nm} \mathrm{SiO}_{2}\right)^{\wedge} 8\right) 115 \mathrm{~nm} \mathrm{HfO} 2385 \mathrm{~nm}$ $\mathrm{SiO}_{2}$, which were the fundamental component of PCGs. The MMLD, four pairs of dielectric bilayers were replaced by the Au layer, was deposited by e-beam evaporation. The reflectivity is shown in Fig. 27. It showed that MMLD and MLD exhibited high and flat reflectivity centered at $1053 \mathrm{~nm}$, especially the ultrabroad bandwidth of MMLD, which verified the effectiveness of broadening the bandwidth. The experimental result showed that the LIDT of MLD and MMLD were all about $5 \mathrm{~J} / \mathrm{cm}^{2} .{ }^{113}$ In 2010, Néauport et al. reported a complete process of MMDG, including design, fabrication, and test. With the goal of high DE and LIDT, to optimize the parameters of the surface relief, the designed grating profile was trapezoidal geometry, instead of traditional rectangle. The MMDG, which was deposited by e-beam evaporation, showed DE reach $96 \%$ for TE polarization centered at $1053 \mathrm{~nm}$, and the LIDT was about $3 \mathrm{~J} / \mathrm{cm}^{2}$ under pulse duration of $500 \mathrm{fs}$, which was similar to that of MDGs. ${ }^{139}$

In 2014, Guan et al. pointed out that the surface relief of $\mathrm{HfO}_{2}$ material showed good bandwidth, but it was difficult to etch. The surface relief of $\mathrm{SiO}_{2}$ material should etch big groove

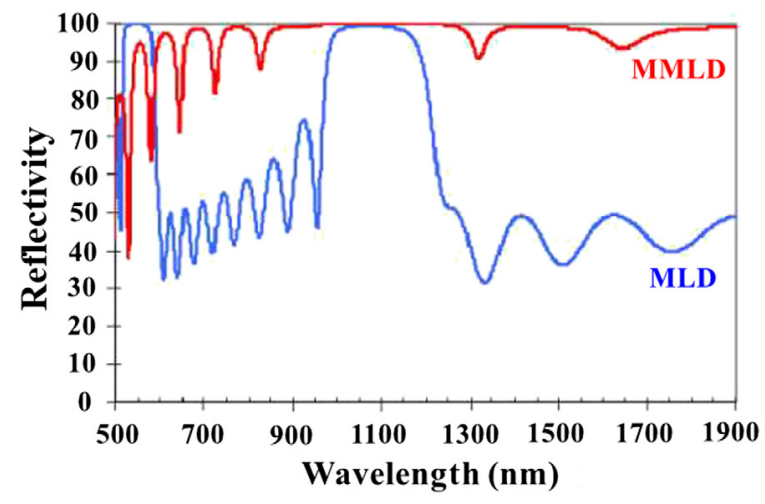

Fig. 27 Reflectivity of the designed MLD and MMLD measured under incidence of 70.6 deg for TE polarization. ${ }^{139}$ 


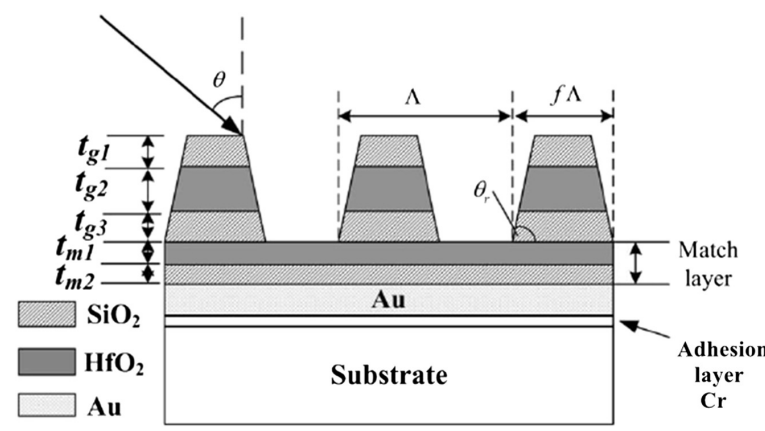

(a)

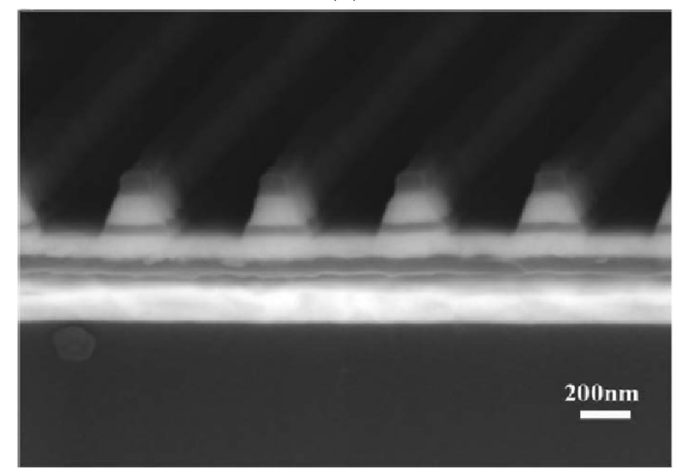

(c)

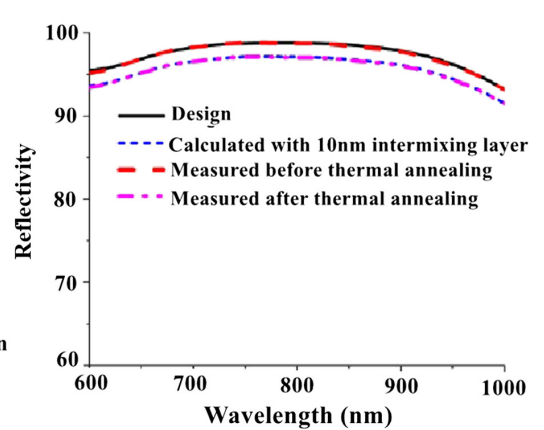

(b)

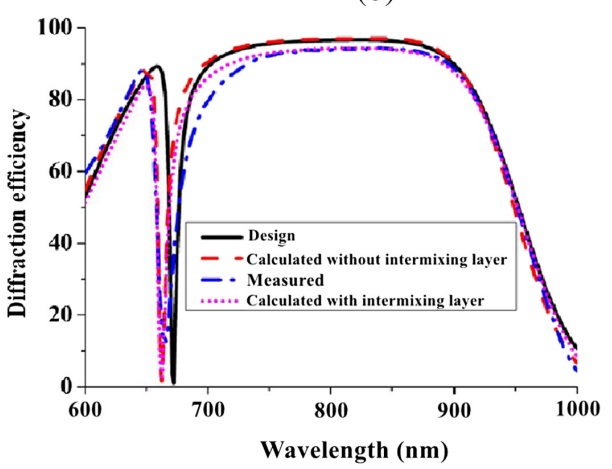

(d)

Fig. 28 (a) Structure of new MMDG. The grating ridge consisted of an $\mathrm{HfO}_{2}$ center layer sandwiched between two $\mathrm{SiO}_{2}$ layers. (b) Reflectivity of MMLD under incidence of 53 deg for TE polarization. The theoretical reflectivity was coincided with the measured over the range of 600 to $800 \mathrm{~nm}$. (c) SEM image of fabricated MMDG. Its duty cycle, period, and slope angle were $0.44,565 \mathrm{~nm}$, and $74 \mathrm{deg}$, respectively. (d) The first-order DE of MMDG under incidence of $53 \mathrm{deg}$ for TE polarization at $800 \mathrm{~nm} .{ }^{155}$

depth and line density, but it had the advantages of high LIDT and easy fabrication. To unite the superiorities of $\mathrm{HfO}_{2}$ and $\mathrm{SiO}_{2}$ materials, a new type of MMDG was designed, as shown in Fig. 28. The grating ridge consisted of one layer $\mathrm{HfO}_{2}$ sandwiched between two layers $\mathrm{SiO}_{2}$. The bandwidth with DE higher than $90 \%$ was $200 \mathrm{~nm}$, and $95 \%$ was $137 \mathrm{~nm}$. The size of fabricated MMDG was $50 \mathrm{~mm} \times 50 \mathrm{~mm}$. The test results showed that the experimental DE was consistent with theoretical simulation, which verified the favorable fabrication tolerance. The LIDT was $0.32 \mathrm{~J} / \mathrm{cm}^{2}$ under $45 \mathrm{fs}$ pulse duration at $800 \mathrm{~nm}$. The optical performance of the MMDG was not the best among the reported MMDGs. However, its performance was well consistent with the designed MMDG, which built the foundations for the functionization and fabrication technology of MMDG. ${ }^{155} \mathrm{Wu}$ et al. investigated the influence of annealing temperature on MMLD. It indicated that the roughness of the MMLD changed slightly after annealing. However, the resistance to chemical cleaning damage improved with the annealing temperature increase, whereas the reflectivity decreased. The MMLD annealed at $250^{\circ} \mathrm{C}$ for $10 \mathrm{~h}$ can be an optimal annealing process for the fabrication of MMDG. ${ }^{191}$ In 2016, Zhang et al. pointed out that the reason for resistance improvement to chemical cleaning damage after annealing was that one transition layer was produced between $\mathrm{Au}$ layer and $\mathrm{SiO}_{2}$ layer, and the transition layer enhanced the adhesion between $\mathrm{Au}$ layer and $\mathrm{SiO}_{2}$ layer and blocked the infiltration of acid solutions. ${ }^{192}$

Chen et al. deeply investigated and explored the fabrication process, including modification of resist masks, the etching speed of different materials, and the cleaning of MMDG. The DE of the fabricated MMDG had a peak DE of $95.1 \%$ at $810 \mathrm{~nm}$ and the maximum bandwidth is $169 \mathrm{~nm}$ with DE higher than $90 \%$, the average DE was $93.71 \%$ under incidence of 53 deg for TE polarization, the LIDT was $0.32 \mathrm{~J} / \mathrm{cm}^{2}$ for $45 \mathrm{fs}$ pulse duration. This was the first particular report for the fabrication process. ${ }^{155,193-195}$ 
Recently, Zou et al. experimentally and theoretically investigated the effect of nodular defect on damage of MMDG. It was found that the initial damages occurred in the grating ridge of the perfect MMDG, whereas initial damages will occur in the district of nodular defect if nodular defect exists. The damage process and the relationship between damage degree and the nodular defect size were theoretically analyzed. It was concluded that local electric field enhancement in nodular defect area would lead to the damage. ${ }^{196} \mathrm{Xu}$ et al. reported a new MMDG with single $\mathrm{Au}$ layer and one silica layer. Its LIDT can reach $0.40 \mathrm{~J} / \mathrm{cm}^{2}$ under $32 \mathrm{fs}$ pulse duration, which was twice more than that of gold-coated gratings. They theoretical predicted that the maximum LIDT of MMDG was $0.60 \mathrm{~J} / \mathrm{cm}^{2} .{ }^{197}$

Although MMDG exhibited high diffraction, broad bandwidth, and high LIDT, the technique was not mature enough to fabricate perfect MMDG due to the existence of metal layer. More efforts should be paid to improve the performance of fabricated MMDGs. ${ }^{198}$

\section{Discussions}

The PCGs are still essential element for generation of ultrahigh intensity laser. And the optical performance requirements are becoming more rigorous. The structures of PCGs are almost perfect after the intensive research. However, it is difficult to improve the optical performance through optimizing the structures. In the past five years, the researchers are focusing on the fabrication technologies and chemical treatment technologies to improve the LIDT.

However, there are still some barriers remaining to overcome to obtain PCGs with better performance. We hold the opinion that there are still two aspects of work to be done in the future. (i) The matching between the designed and fabricated structure. The shape of designed groove is almost standard rectangle. However, it is difficult to etch the rectangle shape, and it is close to trapezoid which leads to the disagreement between theoretical and experimental results. In addition, it is fragile when the duty cycle is too small or the groove depth is too large. The design of PCGs should refer to the fabrication technology, and makes it easy to fabricate. (ii) The improvement of LIDT. The intensity of laser in ever increasing, Consequently, The LIDT should be further improved. The LIDT of ACG cannot still be equated with that of MDG.

\section{Conclusions and Future Prospects}

The CPA technique has opened the door to develop ultrahigh intensity laser, and the performance of PCGs plays a key role in the generation of ultrahigh intensity laser. In the past three decades, significant progress on PCGs has been made. In this paper, we deeply review the progress on ACGs, MDGs and MMDGs in terms of structures, optical performance, and fabrication technology. We believe that it is helpful for the comprehensive understanding on PCGs.

\section{Acknowledgments}

This work was supported by Shandong Provincial Natural Science Foundation, China (Nos. ZR2020QA066 and ZR2020MA087) and the National Natural Science Foundation of China (NSFC) (Nos. 11904213 and 11704226).

\section{References}

1. E. L. Clark et al., "Measurements of energetic proton transport through magnetized plasma from intense laser interactions with solids," Phys. Rev. Lett. 84, 670 (2000).

2. J. Faure et al., "Controlled injection and acceleration of electrons in plasma wakefields by colliding laser pulses," Nature 444, 737-739 (2006).

3. G. Li et al., "Laser channeling in millimeter-scale underdense plasmas of fast-ignition targets," Phys. Rev. Lett. 100, 125002 (2008).

4. C. Hernández-García et al., "Attosecond twisted beams from high-order harmonic generation driven by optical vortices," High Power Laser Sci. Eng. 5, e3 (2017). 
5. I. Y. Skobelev et al., "X-ray absorption spectroscopy study of energy transport in foil targets heated by petawatt laser pulses," Photonics Res. 6, 234-237 (2018).

6. S. G. Fu et al., "Large-energy mode-locked Er-doped fiber laser based on indium selenide as a modulator," Opt. Mater. Express 9, 2662-2671 (2019).

7. G. M. Wang et al., "Decaying evolution dynamics of double-pulse mode-locking," Photonics Res. 6, 825-829 (2018).

8. N. N. Xu et al., "Large-energy passively Q-switched Er-doped fiber laser based on CVD-Bi $\mathrm{Se}_{3}$ as saturable absorber," Opt. Mater. Express 9, 373-383 (2019).

9. P. Maine et al., "Generation of ultrahigh peak power pulses by chirped pulse amplification," IEEE J. Quantum Electron. 24, 398-403 (1988).

10. J. Kmetec, J. J. Macklin, and J. F. Young, "0.5-TW, 125-fs Ti-sapphire laser," Opt. Lett. 16, 1001-1003 (1991).

11. P. Beaud et al., "8-TW 90-fs CrLiSAF laser," Opt. Lett. 18, 1550-1552 (1993).

12. T. Ditmire and M. D. Perry, "Terawatt Cr-LiSrAlF6 laser system," Opt. Lett. 18, 426-428 (1993).

13. M. D. Perry et al., "Petawatt laser pulses," Opt. Lett. 24, 160-162 (1999).

14. M. Aoyama et al., "0.85-PW, 33-fs Ti:sapphire laser," Opt. Lett. 28, 1594-1596 (2003).

15. I. V. Yakovlev, "Stretchers and compressors for ultra-high power laser systems," Quantum Electron. 44, 393-414 (2014).

16. M. Pessot et al., "Chirped-pulse amplification of 100-fsec pulses," Opt. Lett. 14, 797-799 (1989).

17. C. Dorrer et al., "High-contrast optical-parametric amplifier as a front end of high-power laser systems," Opt. Lett. 32, 2143-2145 (2007).

18. K. D. Niu et al., "Passively mode-locked Er-doped fiber laser based on SnS2 nanosheets as a saturable absorber," Photonics Res. 6, 72-76 (2018).

19. H. Kiriyama et al., "High-energy, high-contrast, multiterawatt laser pulses by optical parametric chirped-pulse amplification," Opt. Lett. 32, 2315-2317 (2007).

20. C. Liu et al., "Contrast enhancement in a Ti:sapphire chirped-pulse amplification laser system with a noncollinear femtosecond optical-parametric amplifier," Opt. Lett. 35, 3096-3098 (2010).

21. Z. H. Wang et al., "High-contrast 1.16 PW Ti:sapphire laser system combined with a doubled chirped-pulse amplification scheme and a femtosecond optical-parametric amplifier," Opt. Lett. 36, 3194-3196 (2011).

22. T. J. Yu et al., "Generation of high-contrast, $30 \mathrm{fs}, 1.5 \mathrm{PW}$ laser pulses from chirped pulse amplification Ti:sapphire laser," Opt. Express 20, 10807-10815 (2012).

23. N. N. Xu et al., "Tellurene-based saturable absorber to demonstrate large-energy dissipative soliton and noise-like pulse generations," Nanophotonics 9, 545 (2020).

24. H. N. Zhang et al., "Palladium selenide as a broadband saturable absorber for ultra-fast photonics," Nanophotonics 9, 116 (2020).

25. P. F. Ma et al., "Preparation of high-damage threshold WS2 modulator and its application for generating high-power large-energy bright-dark solitons," Infrared Phys. Technol. 105, 103257 (2020).

26. K. D. Niu et al., "Passively Q-switched erbium-doped fiber laser based on SnS2 saturable absorber," Opt. Mater. Express 7, 3934-3943 (2017).

27. N. Ming et al., "Mode-locked Er-doped fiber laser based on $\mathrm{PbS} / \mathrm{CdS}$ core/shell quantum dots as saturable absorber," Opt. Express 26, 9017-9026 (2018).

28. H. N. Zhang and J. Liu, "Gold nanobipyramids as saturable absorbers for passively Q-switched laser generation in the $1.1 \mu \mathrm{m}$ region," Opt. Lett. 41, 1150-1152 (2016).

29. Y. Chu et al., "High-contrast 2.0 Petawatt Ti:sapphire laser system," Opt. Express 21, 29231-29239 (2013).

30. Y. Chu et al., "High-energy large-aperture Ti:sapphire amplifier for 5 PW laser pulses," Opt. Lett. 40, 5011-5014 (2015).

31. S. Li et al., "High-order dispersion control of 10-petawatt Tisapphire laser facility," Opt. Express 25, 17488-17498 (2017).

32. W. Li et al., "339-J high-energy Ti:sapphire chirped-pulse amplifier for 10-PW laser facility," Opt. Lett. 43, 5681-5684 (2018) 
33. M. D. Perry and G. Mourou, "Terawatt to petawatt subpicosecond lasers," Science 264, 917-924 (1994).

34. D. Strickland and G. Mourou, "Compression of amplified chirped optical pulses," Opt. Commun. 56, 219-221 (1985).

35. Y. X. Jin, K. Yi, and J. D. Shao, "Progress in reflective diffraction gratings used in high power laser system," in OSA Adv. Solid State Lasers Conf., p. ATu2A.30 (2015).

36. N. Bonod and J. Néauport, "Diffraction gratings: from principles to applications in highintensity lasers," Adv. Opt. Photonics 8, 156-159 (2016).

37. E. Gerstner, "Laser physics: extreme light," Nature 446, 16-18 (2007).

38. J. Néauport et al., "Design, optical characterization, and operation of large transmission gratings for the laser integration line and laser megajoule facilities," Appl. Opt. 44, 3143 3152 (2005).

39. H. T. Nguyen et al., "High-efficiency fused-silica transmission gratings," Opt. Lett. 22, 142-144 (1997).

40. H. Ichikawa and K. Minoshima, "Femtosecond laser pulses diffracted by dielectric transmission gratings in the resonance-domain," Opt. Coтmun. 163, 243-251 (1999).

41. J. Nishii, K. Kintaka, and T. Nakazawa, "High-efficiency transmission gratings buried in a fused- $\mathrm{SiO}_{2}$ glass plate," Appl. Opt. 43, 1327-1330 (2004).

42. T. Clausnitzer et al., "Highly efficient transmission gratings in fused silica for chirpedpulse amplification systems," Appl. Opt. 42, 6934-6938 (2003).

43. Y. X. Tang et al., "Transmission grating stretcher for contrast enhancement of high power lasers," Opt. Express 22, 29363-29374 (2014).

44. J-K. Rhee et al., "Chirped-pulse amplification of 85 -fs pulses at $250 \mathrm{kHz}$ with third-order dispersion compensation by use of holographic transmission gratings," Opt. Lett. 19, 1550-1552 (1994).

45. T. J. Kessler et al., "Demonstration of coherent addition of multiple gratings for highenergy chirped-pulse-amplified lasers," Opt. Lett. 29, 635-637 (2004).

46. T. J. Zhang, M. Yonemura, and Y. Kato, "Optical design of an array-grating compressor for high-power laser pulses," Fusion Eng. Design 44, 127-131 (1999).

47. M. Hornung et al., "Alignment of a multigrating mosaic compressor in a PW-class CPA-laser," Proc. SPIE 5962, 59622K (2005).

48. N. Blanchot et al., "Synthetic aperture compression scheme for a multipetawatt highenergy laser," Appl. Opt. 45, 6013-6021 (2006).

49. A. Cotel et al., "Phased-array grating compression for high-energy chirped pulse amplification lasers," Opt. Express 15, 2742-2752 (2007).

50. M. C. Rushford et al., "Split-aperture laser pulse compressor design tolerant to alignment and line-density differences," Opt. Lett. 33, 1902-1904 (2008).

51. N. Blanchot et al., "1.15 PW-850 J compressed beam demonstration using the PETAL facility," Opt. Express 25, 16957-16970 (2017).

52. N. Blanchot et al., "Experimental demonstration of a synthetic aperture compression scheme for multi-petawatt high-energy lasers," Opt. Express 18, 10088-10097 (2010).

53. D. Maystre, "Photonic crystal diffraction gratings," Opt. Express 8, 209-216 (2001).

54. N. Bonod and J. Néauport, "Design of a full-silica pulse-compression grating," Opt. Lett. 33, 458-460 (2008).

55. A. J. Waddie, M. J. Thomson, and M. R. Taghizadeh, "Comparison of one- and twodimensional dielectric reflector geometries for high-energy laser pulse compression," Opt. Lett. 30, 991-993 (2005).

56. A. Tishchenko and V. Sychugov, "High grating efficiency by energy accumulation in a leaky mode," Opt. Quantum Electron. 32, 1027-1031 (2000).

57. N. Destouches et al., "99\% efficiency measured in the -1st order of a resonant grating," Opt. Express 13, 3230-3235 (2005).

58. M. Flury, A. V. Tishchenko, and O. Parriaux, "The leaky mode resonance condition ensures $100 \%$ diffraction efficiency of mirror-based resonant gratings," J. Lightwave Technol. 25, 1870-1878 (2007).

59. J. R. Marciante and D. H. Raguin, "High-efficiency, high-dispersion diffraction gratings based on total internal reflection," Opt. Lett. 29, 542-544 (2004). 
60. Q. Y. Bi et al., "Design of ultrabroadband internal reflection gratings with high efficiency," Opt. Lett. 36, 1431-1433 (2011).

61. B. Wang, "High-efficiency two-port beam splitter of total internal reflection fused-silica grating," J. Phys. B: At. Mol. Opt. Phys. 44, 065402 (2011).

62. W. Jia et al., "Miniature pulse compressor of deep-etched gratings," Appl. Opt. 47, 60586063 (2008).

63. A. Villamarin et al., "Pulse compression with volume holographic transmission gratings recorded in Slavich PFG-04 emulsion," Proc. SPIE 7430, 74300Y (2009).

64. P-A. Blanche et al., "Volume phase holographic gratings: large size and high diffraction efficiency," Opt. Eng. 43, 2603-2612 (2004).

65. B. Wang et al., "Connecting-layer-based polarizing beam splitter grating with high efficiency for both TE and TM polarizations," Opt. Laser Technol. 44, 2145-2148 (2012).

66. B. Wang et al., "Enhancement of the efficiency based on a sandwiched grating for separating polarized beams," IEEE Photonics Technol. Lett. 24, 1513-1515 (2012).

67. H. T. Li and B. Wang, "Two-port connecting-layer-based sandwiched grating by a polarization-independent design," Sci. Rep. 7, 1309 (2017).

68. B. Wang et al., "Metal-mirror-based grating for high-efficiency element in dense wavelength division multiplexing," Opt. Commun. 285, 4599-4602 (2012).

69. B. Wang et al., "Deep-etched high-spatial-frequency metal-based sandwiched grating for reflective dual-function beam splitter," Opt. Laser Technol. 49, 81-85 (2013).

70. B. Wang et al., "Metal-based phase grating for high-efficiency polarizing beam splitter," Opt. Commun. 296, 149-152 (2013).

71. B. Wang, "Suppression of the zeroth order by a mixed metal dielectric grating," IEEE Photonics J. 8, 7801706 (2016).

72. B. Wang et al., "Two-layer dielectric grating as two-port beam splitter," IEEE Photonics Technol. Lett. 25, 863-866 (2013).

73. B. Wang et al., "Diffractive polarizing beam splitter of two-layer grating for operation in reflection," Opt. Commun. 311, 307-310 (2013).

74. B. Wang et al., "Modal analysis and numerical design of wideband two-layer grating with low polarization dependence," Opt. Commun. 306, 74-77 (2013).

75. B. Wang et al., "Reflection-reduced two-layer grating with nearly $100 \%$ diffraction efficiency," IEEE Photonics Technol. Lett. 26, 501-503 (2014).

76. B. Wang et al., "Performance investigation of incident bandwidth based on reflective two-layer grating with high efficiency," Opt. Laser Technol. 56, 372-374 (2014).

77. H. T. Li and B. Wang, "Three-layer grating with the enhanced efficiency and angular bandwidth," IEEE Photonics J. 9, 1-7 (2017).

78. NobelPrize. org, "The Nobel Prize in Physics 2018," https://www.nobelprize.org/prizes/ physics/2018/summary/ (2018).

79. W. J. Kong, Optimization, Manufacture and Analysis of Multi-Layer Dielectric for Pulse Compressor Gratings, Shanghai Institute of Optics and Fine Mechanics, Chinese Academy of Sciences, Shanghai (2006).

80. M. G. Moharam and T. K. Gaylord, "Rigorous coupled-wave analysis of planar-grating diffraction," J. Opt. Soc. Am. 71(7), 811-818 (1981).

81. M. G. Moharam and T. K. Gaylord, "Coupled-wave analysis of reflection gratings," Appl. Opt. 20(2), 240-244 (1981).

82. M. G. Moharam and T. K. Gaylord, "Chain-matrix analysis of arbitrary-thickness dielectric reflection gratings," J. Opt. Soc. Am. 72(2), 187-190 (1982).

83. M. G. Moharam and T. K. Gaylord, "Diffraction analysis of dielectric surface-relief gratings," J. Opt. Soc. Am. 72(10), 1385-1392 (1982).

84. W. E. Baird, M. G. Moharam, and T. K. Gaylord, "Diffraction characteristic of planar absorption gratings," Appl. Phys. B 32(1), 15-20 (1983).

85. M. G. Moharam and T. K. Gaylord, "Rigorous coupled-wave analysis of metallic surfacerelief gratings," J. Opt. Soc. Am., 3(11), 1780-1787 (1986).

86. M. G. Moharam et al., "Formulation for stable and efficient implementation of the rigorous coupled-wave analysis of binary gratings," J. Opt. Soc. Am. 12(5), 1068-1086 (1995). 
87. L. Li, "A modal analysis of lamellar diffraction gratings in conical mountings," J. Mod. Opt. 40(4), 553-573 (1993).

88. L. Li, "Multilayer modal method for diffraction gratings of arbitrary profile, depth, and permittivity," J. Opt. Soc. Am. A 10(12), 2581-2591 (1993).

89. L. Mashev and S. Tonchev, "Formation of holographic diffraction gratings in photoresist," Appl. Phys. A 26, 143-149 (1981).

90. K. Yokomori, "Dielectric surface relief gratings with high diffraction efficiency," Appl. Opt. 23, 2303-2310 (1984).

91. R. R. Hershey and E. N. Leith, "Grating interferometers for producing large holographic gratings," Appl. Opt. 29, 937-943 (1990).

92. E. K. Popov, L. V. Tsonev, and M. L. Sabeva, "Technological problems in holographic recording of plane gratings," Opt. Eng. 31, 2168-2173 (1992).

93. M. Sabeva, E. Popov, and L. Tsonev, "Reflection gratings in the visible region-efficiency in nonpolarized light," Opt. Commun. 100, 39-42 (1993).

94. L. L. Wang et al., "Evaluation of femtosecond laser damage to gold pulse compression gratings fabricated by magnetron sputtering and e-beam evaporation," Appl. Opt. 56, 3087-3095 (2017).

95. B. N. Chichkov et al., "Femtosecond, picosecond and nanosecond laser ablation of solids," Appl. Phys. A 63, 109-115 (1996).

96. B. C. Stuart et al., "Nanosecond-to-femtosecond laser-induced breakdown in dielectrics," Phys. Rev. B 53, 1749-1761 (1996).

97. J. Jasapara et al., "Femtosecond laser pulse induced breakdown in dielectric thin films," Phys. Rev. B 63, 045117 (2001).

98. M. Mero et al., "Scaling laws of femtosecond laser pulse induced breakdown in oxide films," Phys. Rev. B 71, 115109 (2005).

99. X. F. Jing et al., "Calculation of femtosecond pulse laser induced damage threshold for broadband antireflective microstructure arrays," Opt. Express 17, 24137-24152 (2009).

100. M. Jupé et al., "Calculations and experimental demonstration of multi-photon absorption governing fs laser-induced damage in titania," Opt. Express 17, 12269-12278 (2009).

101. L. Gallais et al., "Transient interference implications on the subpicosecond laser damage of multidielectrics," Appl. Phys. Lett. 97, 051112 (2010).

102. S. Hocquet, J. Néauport, and N. Bonod, "The role of electric field polarization of the incident laser beam in the short pulse damage mechanism of pulse compression gratings," Appl. Phys. Lett. 99, 061101 (2011).

103. X. B. Cheng et al., "The effect of an electric field on the thermomechanical damage of nodular defect in dielectric multilayer coatings irradiated by nanosecond laser pulses," Light: Sci. Appl. 2, e80 (2013).

104. B. Chimier et al., "Damage and ablation thresholds of fused-silica in femtosecond regime," Phys. Rev. B 84, 094104 (2011).

105. C. Danson et al., "Petawatt class lasers worldwide," High Power Laser Sci. Eng. 3, e3 (2015).

106. P. Poole et al., "Femtosecond laser damage threshold of pulse compression gratings for petawatt scale laser systems," Opt. Express 21, 26341-26351 (2013).

107. D. N. Papadopoulos et al., "The Apollon 10 PW laser: experimental and theoretical investigation of the temporal characteristics," High Power Laser Sci. Eng. 4, e34 (2016).

108. V. D. Vinokurova et al., "Metallised holographic diffraction gratings with the enhanced radiation resistance for laser pulse compression systems," Quantum Electron. 35, 569572 (2005).

109. R. D. Boyd et al., "High-efficiency metallic diffraction gratings for laser applications," Appl. Opt. 34, 1697-1706 (1995).

110. B. C. Stuart et al., "Optical ablation by high-power short-pulse lasers," J. Opt. Soc. Am. B 13, 459-468 (1996).

111. J. A. Britten et al., "Universal grating design for pulse stretching and compression in the 800-1100-nm range," Opt. Lett. 21, 540-542 (1996).

112. M. D. Perry et al., "High-efficiency multilayer dielectric diffraction gratings," Opt. Lett. 20, 940-942 (1995). 
113. L. F. Li and J. Hirsh, "All-dielectric high-efficiency reflection gratings made with multilayer thin-film coatings," Opt. Lett. 20, 1349-1351 (1995).

114. J. A. Britten et al., "High-efficiency dielectric multilayer gratings optimized for manufacturability and laser damage threshold," Proc. SPIE 2714, 511-520 (1996).

115. B. W. Shore et al., "Design of high-efficiency dielectric reflection gratings," J. Opt. Soc. Am. A 14, 1124-1136 (1997).

116. K. Hehl et al., "High-efficiency dielectric reflection gratings: design, fabrication, and analysis," Appl. Opt. 38, 6257-6271 (1999).

117. H. B. Wei and L. F. Li, "All-dielectric reflection gratings: a study of the physics mechanism for achieving high efficiency," Appl. Opt. 42, 6255-6260 (2003).

118. W. J. Kong et al., "Design and properties analysis of multi-layer dielectric used in pulse compressor gratings," Optik 116, 325-330 (2005).

119. W. J. Kong et al., "Design and character analysis of multilayer dielectric film used in pulse compressed gratings," Acta Opt. Sin. 25, 701-706 (2005).

120. S. J. Liu et al., "Optimization of near-field optical field of multilayer dielectric gratings for pulse compressor," Opt. Commun. 267, 50-57 (2006).

121. S. J. Liu et al., "Near-field optical property of multi-layer dielectric gratings for pulse compressor," Acta Phys. Sin. 55, 4588-4594 (2006).

122. S. J. Liu et al., "Optimization of thin film design for multilayer dielectric grating," Appl. Surf. Sci. 253, 3642-3648 (2007).

123. J. Néauport et al., "Effect of electric field on laser induced damage threshold of multilayer dielectric gratings," Opt. Express 15, 12508-12522 (2007).

124. J. P. Wang et al., "Analysis of restriction factors of widening diffraction bandwidth of multilayer dielectric grating," Chin. Phys. B. 19, 104201 (2010).

125. J. P. Wang et al., "Design of shallow etched multilayer dielectric grating with -1 st order high diffraction efficiency," Chin. Opt. Lett. 8, 29-31 (2010).

126. J. P. Wang et al., "Optimization design of an ultrabroadband, high-efficiency, all-dielectric grating," Opt. Lett. 35, 187-189 (2010).

127. C. T. Chang and J. L. Bjorkstam, "Amplitude hologram efficiencies with arbitrary modulation depth, based upon a realistic photographic film model," J. Opt. Soc. Am. 67, 1160-1164 (1977).

128. A. Svakhin et al., "Efficient diffraction elements for TE-polarized waves," Sov. Phys. Tech. Phys. 36, 1038-1040 (1991).

129. A. S. Svakhin, V. Sychugov, and A. Tikhomirov, "Diffraction gratings with high optical strength for laser resonators," Quantum Electron. 24, 233-235 (1994).

130. J. A. Britten et al., "Multilayer dielectric gratings for petawatt-class laser systems," Proc. SPIE 5273, 1-7 (2004).

131. A. Reichart et al., "CPA compression gratings with improved damage performance," Proc. SPIE 4347, 521-527 (2001).

132. M. D. Perry et al., "Ultrashort-pulse laser machining of dielectric materials," J. Appl. Phys. 85, 6803 (1999).

133. C. P. J. Barty et al., "An overview of LLNL high-energy short-pulse technology for advanced radiography of laser fusion experiments," Nucl. Fusion 44, S266-S275 (2004).

134. A. Cotel et al., "Characterization of multilayer dielectric gratings for petawatt-class lasers," in Quantum Electron. and Laser Sci. Conf. (QELS'05), Vol. 3, 2038-2040 (2005).

135. A. Bunkowski et al., "Optical characterization of ultrahigh diffraction efficiency gratings," Appl. Opt. 45, 5795-5799 (2006).

136. Y. P. Dai et al., "Multilayer dielectric gratings for ultrashort pulse compressor," Proc. SPIE 6403, 64031B (2006).

137. P. P. Lu et al., "Precise diffraction efficiency measurements of large-area greater-than-99\%efficient dielectric gratings at the Littrow angle," Opt. Lett. 34, 1708-1710 (2009).

138. H. Y. Guan et al., "Broadband trapeziform multilayer dielectric grating for femtosecond pulse compressor: design, fabrication, and analysis," Laser Phys. 23, 115301 (2013).

139. S. Palmier et al., "High reflection mirrors for pulse compression gratings," Opt. Express 17, 20430-20439 (2009). 
140. N. Bonod and J. Néauport, "Optical performance and laser induced damage threshold improvement of diffraction gratings used as compressors in ultrahigh intensity lasers," Opt. Commun. 260, 649-655 (2006).

141. J. Néauport and N. Bonod, "Pulse compression gratings for the PETAL project: a review of various technologies," Proc. SPIE 7132, 71320D (2008).

142. J. Néauport et al., "Mixed metal dielectric gratings for pulse compression," Opt. Express 18, 23776-23783 (2010).

143. K. He et al., "High-spectral-resolution characterization of broadband high-efficiency reflection gratings," Appl. Opt. 52, 653-658 (2013).

144. J. Qiao, J. Papa, and X. Liu, "Spatio-temporal modeling and optimization of a deformablegrating compressor for short high-energy laser pulses," Opt. Express 23, 25923-25934 (2015).

145. M. Flury et al., "High-efficiency wide-band metal-dielectric resonant grating for 20 -fs pulse compression," J. Eur. Opt. Soc. Rapid Publ. 2, 07024 (2007).

146. W. J. Kong et al., "Diffraction property of broadband metal multi-layer dielectric gratings based on rigorous coupled-wave analysis," Acta Phys. Sin. 60, 114214 (2011).

147. W. J. Kong et al., "Design and diffraction property of broad waveband high-diffractionefficiency grating for chirped pulse compressor," Acta Opt. Sin. 31, 1005001 (2011).

148. W. J. Kong et al., "Optimization and tolerance of ultra-broadband metal multi-layer dielectric gratings for femtosecond lasers," J. Optoelectron. Laser 22, 1769-1773 (2011).

149. W. J. Kong et al., "Broadband and high efficiency metal-multilayer dielectric grating based on non-quarter wave coatings as reflective mirror for $800 \mathrm{~nm}$," J. Mod. Opt. 59, 1680-1685 (2012).

150. W. F. Zhang et al., "Broadband and high efficiency metal multi-layer dielectric grating based on non-quarter wave coatings as reflective mirror," Chin. Phys. B 21, 094218 (2012).

151. A. D. Hu et al., "Modal analysis of high-efficiency wideband reflective gratings," J. Opt. 14, 055705 (2012).

152. S. H. Wang et al., "Broadband and high-efficiency metal-multilayer dielectric grating centered at $800 \mathrm{~nm}$ based on non-quarter wave coatings as reflective mirror," Proc. SPIE 8786, 878627 (2013).

153. J. P. Wang et al., "Design and analysis of broadband high-efficiency pulse compression gratings," Appl. Opt. 49, 2969-2978 (2010).

154. H. Y. Guan et al., "Near-field optical properties of wide bandwidth metal multi-layer dielectric gratings for pulse compressor," Appl. Phys. B: Lasers Opt. 114, 557-565 (2013).

155. H. Y. Guan et al., "High-efficiency, broad-bandwidth metal/multilayer-dielectric gratings," Opt. Lett. 39, 170-173 (2014).

156. A. D. Hu et al., "Polarization-independent wideband mixed metal dielectric reflective gratings," Appl. Opt. 51, 4902-4906 (2012).

157. H. P. Huang et al., "Effects of substrate on the femtosecond laser-induced damage properties of gold films," Opt. Mater. 81, 115-121 (2018).

158. B. Wang and L. Gallais, "A theoretical investigation of the laser damage threshold of metal multi-dielectric mirrors for high power ultrashort applications," Opt. Express 21, 1469814711 (2013).

159. Y. Kitagawa et al., "Prepulse-free petawatt laser for a fast ignitor," IEEE J. Quantum Electron. 40, 281-293 (2004).

160. C. Danson et al., "Vulcan petawatt: design, operation and interactions at $5 * 1020 \mathrm{~W} \mathrm{~cm}^{-2}$," Laser Part. Beams 23, 87-93 (2005).

161. C. Li et al., "Design and fabricate large scale gold coated pulse compression grating for Ti:sapphire laser," Proc. SPIE 9266, 92661K (2014).

162. B. Muhutijiang et al., "Design and fabrication of sine-top broadband gold-coated gratings," Opt. Eng. 54, 105109 (2015).

163. H. P. Huang et al., "Ultrashort pulses-driven dynamics of blisters in Au-coated gratings," Opt. Mater. 72, 130-135 (2017).

164. J. B. Oliver et al., "Thin-film design for multilayer diffraction gratings," Proc. SPIE 5991, 59911A (2005). 
165. B. Ashe et al., "Evaluation of cleaning methods for multilayer diffraction gratings," Proc. SPIE 6403, 640300 (2007).

166. B. Ashe et al., "Optimizing a cleaning process for multilayer-dielectric-(MLD) diffraction grating," Proc. SPIE 6720, 67200N (2007).

167. W. J. Kong et al., "Laser induced damage thresholds of multilayer dielectric gratings and multilayer dielectric mirrors," Chin. J. Lasers 33, 552-556 (2006).

168. W. J. Kong et al., "Design of multilayer dielectric grating for femtosecond laser," Acta Phys. Sin. 55, 1143-1147 (2006).

169. W. J. Kong et al., "Multi-layer dielectric film for pulse compressed gratings," Acta Photon. Sin. 35, 84-88 (2006).

170. N. Lyndin et al., "Design and fabrication of an all-dielectric grating with top-hat high diffraction efficiency over a broad spectral range," J. Eur. Opt. Soc. Rapid Publ. 2, 07019 (2007).

171. S. J. Liu et al., "Characteristics of high reflection mirror with $\mathrm{SiO}_{2}$ top layer for multilayer dielectric grating," J. Phys. D: Appl. Phys. 40, 3224-3228 (2007).

172. S. J. Liu et al., "Performance of multilayer dielectric grating irradiated by ultrashort optical pulse," Acta Phys. Sin. 56, 4542-4549 (2007).

173. Y. Liu et al., "Reactive ion beam etching of multilayer diffraction gratings with $\mathrm{SiO}_{2}$ as the top layer," Proc. SPIE 6832, 683220 (2007).

174. X. D. Xu et al., "Ion beam etching for multilayer dielectric pulse compressor gratings with top layers of $\mathrm{HfO}_{2}$, , Acta Phys. Sin. 62, 234202 (2013).

175. W. J. Kong et al., "Effect of electric field enhancement on laser induced damage morphology of multilayer dielectric grating," Acta Photonics Sin. 38, 298-301 (2009).

176. D. H. Martz et al., "Large area high efficiency broad bandwidth $800 \mathrm{~nm}$ dielectric gratings for high energy laser pulse compression," Opt. Express 17, 23809-23816 (2009).

177. F. Y. Kong et al., "Effect of pulse duration on laser induced damage threshold of multilayer dielectric gratings,” Proc. SPIE 8530, 85300L (2012).

178. F. Y. Kong et al., "Femtosecond laser damage of broadband pulse compression gratings," Chin. Opt. Lett. 11, 102302 (2013).

179. F. Y. Kong et al., "Investigation of fs-laser induced damage on high reflection mirrors used for 800-nm broadband pulse compression gratings," Opt. Laser Technol. 54, 45-49 (2013).

180. F. Y. Kong et al., "Femtosecond laser damage of all-dielectric pulse compression gratings," Laser Phys. 24, 106101 (2014).

181. F. Y. Kong et al., "Influence of horizontal damage size of grating ridge on the optical properties of multilayer dielectric gratings," Appl. Opt. 53, 4859-4864 (2014).

182. F. Y. Kong et al., "Femtosecond laser induced damage of pulse compression gratings," Opt. Laser Technol. 97, 339-345 (2017).

183. M. Sozet et al., "Sub-picosecond lasler damage growth on high reflective coatings for high power applications," Opt. Express 25, 25767-25781 (2017).

184. Y. F. Hao et al., "Asymmetrical damage growth of multilayer dielectric gratings induced by picosecond laser pulses," Opt. Express 26, 8791-8804 (2018).

185. S. M. Gracewski et al., "Simulation of internal stress waves generated by laser-induced damage in multilayer dielectric gratings," Opt. Express 26, 18412-18422 (2018).

186. H. T. Nguyen, C. C. Larson, and J. A. Britten, "Improvement of laser damage resistance and diffraction efficiency of multilayer dielectric diffraction gratings by HF-etchback linewidth tailoring," Proc. SPIE 7842, 78421H (2010).

187. S. B. Chen et al., "Wet-cleaning of contaminants on the surface of multilayer dielectric pulse compressor gratings by the Piranha solution," Proc. SPIE 7655, 765522 (2010).

188. X. L. Ge et al., "Plasma cleaning of compressed grating in chirped pulse femtosecond laser amplifier," Chin. J. Laser 39, 0402006 (2012).

189. H. P. Howard et al, "Improving the performance of high laser damage threshold, multilayer dielectric pulse compression gratings through low temperature chemical cleaning," Appl. Opt. 52, 1682-1692 (2013).

190. F. Canova et al., "High-efficiency, broad band, high-damage threshold high-index gratings for femtosecond pulse compression," Opt. Express 15, 15324-15334 (2007). 
191. J. B. Wu et al., "Effect of annealing temperature on metal/dielectric multilayers for fabricating broadband pulse compression gratings," J. Inorg. Mater. 29, 1087-1092 (2014).

192. H. Zhang et al., "Influence of annealing on interface diffusion and anti-chemical-cleaning property of metal-dielectric multilayer films," Chin. J. Lasers 43, 1003002 (2016).

193. H. Chen et al., "Fabrication of broadband, high-efficiency, metal-multilayer-dielectric gratings," Opt. Commun. 329, 103-108 (2014).

194. H. Chen, Broadband, High-Efficiency Gratings for Laser Pulse Compression: MetalMultilayer Dielectric Gratings and Metal-Multilayer-Dielectric Coated Gratings, Tsinghua University, Beijing (2015).

195. H. Chen, "Metal/multilayer-dielectric coated gratings for chirped pulse amplification laser system," Proc. SPIE 9271, 92711C (2014).

196. X. Zou "Influence of nodular defect size on metal dielectric mixed gratings for ultra-short ultra-high intensity laser system," Opt. Mater. 91, 177-182 (2019).

197. J. Xu, "Metal dielectric gratings with high femtosecond laser damage threshold of twice as much as that of traditional gold gratings," Opt. Lett. 44, 2871-2874 (2019).

198. Y. Su, "Exploring surface sensitivity of Rayleigh anomaly in metal/dielectric multilayer gratings," Opt. Express 27, 14152-14162 (2019).

Wenfei Zhang received his BS degree in applied physics in 2009 and his MS degree in condensed physics in 2012 from Qingdao University, and his PhD in electric science and technology from Xi' an Jiaotong University, Xi' an, China. His current works are the laser technique, photoelectric properties, and sensing applications of two-dimensional materials.

Weijin Kong received his $\mathrm{PhD}$ in optics engineering in 2006 from Shanghai Institute of Optics and Fine Mechanics, Chinese Academy of Sciences, Shanghai, China. His current work is the laser technique and thin films.

Guomei Wang received her $\mathrm{PhD}$ in optics from Xi'an Institute of Optics and Precision Mechanics, Chinese Academy of Sciences, Xi'an, China. Her current work is the fiber laser technique.

Fei Xing received his BS degree in material physics and chemistry in 2005 from Hebei University of Technology and his PhD in optics in 2014 from Nankai University. He is a postdoctoral researcher at Shenzhen University. His current research interests include twodimensional thin films (graphene, black phosphorus, and molybdenum disulfide) and optical properties of graphene and its applications.

Fang Zhang received her PhD from Shandong University, Jinan, China. Her current research interest is non-linear optics.

Huanian Zhang received his PhD from Shandong University, Jinan, China. His current research interest is the ultrafast fiber laser and its application.

Shenggui Fu received his MS and $\mathrm{PhD}$ degrees from Nankai University, Tianjin, China. He is a postdoctoral researcher at Nankai University. His current research interests include the laser technology and optical fiber photonics. 